OPEN ACCESS

Edited by:

Sergio Lanteri,

University of Turin, Italy

Reviewed by:

Giampiero Valè,

Cereal Research Centre, CRA-CER,

Yongaing Jiao, Oil Crops Research Institute (CAAS),

China

*Correspondence:

Aiping Zheng

aipingzh@163.com

Specialty section:

This article was submitted to Crop Science and Horticulture,

a section of the journal

Frontiers in Plant Science

Received: 01 March 2017

Accepted: 31 July 2017

Published: 17 August 2017

Citation:

Zhang J, Chen L, Fu C, Wang L, Liu H, Cheng Y, Li S, Deng Q,

Wang S, Zhu J, Liang Y, Li P and

Zheng A (2017) Comparative

Transcriptome Analyses of Gene Expression Changes Triggered by Rhizoctonia solani AG1 IA Infection in Resistant and Susceptible Rice Varieties. Front. Plant Sci. 8:1422. doi: 10.3389/fpls.2017.01422

\section{Comparative Transcriptome Analyses of Gene Expression Changes Triggered by Rhizoctonia solani AG1 IA Infection in Resistant and Susceptible Rice Varieties}

\author{
Jinfeng Zhang 1,2,3, Lei Chen 1,2,3, Chenglin Fu'1,2,3, Lingxia Wang 1,2,3, Huainian Liu'1,2,3, \\ Yuanzhi Cheng ${ }^{1,2,3}$, Shuangcheng Li $^{1,2,3}$, Qiming Deng ${ }^{1,2,3}$, Shiquan Wang ${ }^{1,2,3}$, Jun Zhu ${ }^{1,2,3}$, \\ Yueyang Liang ${ }^{1,2,3}$, Ping $\mathrm{Li}^{1,2,3}$ and Aiping Zheng ${ }^{1,2,3 *}$ \\ ${ }^{1}$ Rice Research Institute, Sichuan Agricultural University, Chengdu, China, ${ }^{2}$ State Key Laboratory of Hybrid Rice, Sichuan \\ Agricultural University, Chengdu, China, ${ }^{3}$ Key Laboratory of Sichuan Crop Major Disease, Sichuan Agricultural University, \\ Chengdu, China
}

Rice sheath blight, caused by Rhizoctonia solani, is one of the most devastating diseases for stable rice production in most rice-growing regions of the world. Currently, studies of the molecular mechanism of rice sheath blight resistance are scarce. Here, we used an RNA-seq approach to analyze the gene expression changes induced by the AG1 IA strain of $R$. solani in rice at 12, 24, 36, 48, and $72 \mathrm{~h}$. By comparing the transcriptomes of TeQing (a moderately resistant cultivar) and Lemont (a susceptible cultivar) leaves, variable transcriptional responses under control and infection conditions were revealed. From these data, 4,802 differentially expressed genes (DEGs) were identified. Gene ontology and pathway enrichment analyses suggested that most DEGs and related metabolic pathways in both rice genotypes were common and spanned most biological activities after AG1 IA inoculation. The main difference between the resistant and susceptible plants was a difference in the timing of the response to AG1 IA infection. Photosynthesis, photorespiration, and jasmonic acid and phenylpropanoid metabolism play important roles in disease resistance, and the relative response of disease resistance-related pathways in TeQing leaves was more rapid than that of Lemont leaves at $12 \mathrm{~h}$. Here, the transcription data include the most comprehensive list of genes and pathway candidates induced by AG1 IA that is available for rice and will serve as a resource for future studies into the molecular mechanisms of the responses of rice to $A G 1 \mathrm{IA}$.

Keywords: rice, Rhizoctonia solani AG1 IA, RNA-seq, differentially expressed gene, defense response

\section{INTRODUCTION}

Due to their growth characteristics, plants are constantly exposed to different environmental stresses throughout their life cycle, and pathogen infection is a leading constraint on plant growth and productivity (Dodds and Rathjen, 2010). Rice (Oryza sativa L.), as the most important food crop for human consumption, provides a stable food supply for more than half of the 
world's population. Recently, along with climate change, diseases caused by Rhizoctonia solani have severely limited rice production, leading to drastic economic losses each year that threaten food security (Anderson et al., 2004; Marchetti and Bollich, 1991; Lee et al., 2006). R. solani is a necrotrophic fungus, and its hyphae grow in close association with host surfaces, forming typical lobate appressoria that can give rise to hyphal aggregates known as infection cushions (Marshall and Rush, 1980). The fungus kills host cells prior to infection by deploying toxins and enzymes that induce cell death (Vidhyasekaran et al., 1997; Brooks, 2007). Despite the huge global losses in rice yield caused by sheath blight, only limited information is available regarding effective control of this disease. In recent years, successful attempts have been made to understand the responses of various rice lines to $R$. solani infection, which have been classified as partial resistance mechanisms but are also referred to as incomplete, quantitative, or horizontal resistance mechanisms (Liu et al., 2013). So far, no rice variety has been found to be highly resistant to $R$. solani, and resistance is conferred solely by the additive effect of non-race-specific resistance QTLs (Pinson et al., 2005; Liu et al., 2009). Control strategies have mainly relied on the application of fungicides at the onset of disease. Our knowledge of the intriguing plantnecrotrophic pathogen interaction established between $R$. solani and rice is still very limited. Therefore, dissecting the molecular mechanism of rice sheath blight resistance would be an important step toward developing novel and effective strategies to control diseases.

As the research area of host and pathogen genomics has rapidly expanded, new technology has provided insights into the processes involved in pathogenesis, host-defense responses, and changes to the structure and composition of the genome associated with diverse host-pathogen interactions (Lai and Mengiste, 2013; Okubara et al., 2014). To date, only a few studies have been conducted to analyze the genome-wide expression profiles of rice after $R$. solani infection and the pathogenic molecular mechanism of pathogens. Venu et al. (2007) detected numerous up- and down-regulated rice genes after infection with $R$. solani using SAGE and microarray analysis. Zhao et al. (2008) found fifty unique cDNA clones and assigned them to five functional categories that had not previously been identified as induced in response to pathogens. Silva et al. (2012) used wholegenome sequencing to identify a total of 333 nsSNPs in resistant lines that were absent in the susceptible group. More than 200 genes with selected nsSNPs were assigned to 42 categories based on gene family/gene ontology. Zheng et al. (2013) identified 25 candidate pathogen effectors based on their functionality and evolution, and three were validated to trigger crop defense responses with the draft genome sequence of $R$. solani AG1 IA that was assembled. Hence, research has revealed that pathogenic determinants exhibit exclusive expression patterns during host infection. Although previous studies have investigated the effects of $R$. solani infection, the molecular mechanisms related to the defense responses of rice against $R$. solani exposure remain poorly understood.

The development and application of second-generation sequencing technology, with its high-throughput ability and low cost, have greatly promoted genomic analysis and gene function research (Wang et al., 2009; Jain, 2012). In recent years, numerous pathogen stress-response genes have been identified in Arabidopsis thaliana (Ditt et al., 2006), O. sativa (Bagnaresi et al., 2012), Zea mays (Skibbe et al., 2010), and Triticum aestivum (Yang et al., 2015), and pathogen resistance mechanisms have been explored. To develop a better understanding of the molecular-level variations among rice cultivars after $R$. solani infection, we analyzed the leaf transcriptomes of the rice cultivars TeQing and Lemont after inoculation with AG1 IA. TeQing, a moderately resistant standard rice genotype, and Lemont, a susceptible genotype, were analyzed at 12, 24, 36, 48, and $72 \mathrm{~h}$ after AG1 IA inoculation, and Illumina RNA-seq, bioinformatics, qRT-PCR, and transcriptome expression analyses were performed to identify differentially expressed genes (DEGs) and detail how rice responds to AG1 IA. Here, we investigated how these two rice cultivars induce resistance or susceptibility to $R$. solani AG1 IA and whether this response is based on the differential expression of genes involved in different resistanceassociated metabolic pathways. To our knowledge, this is the first study to use comparative transcriptome profiling analysis to investigate gene expression patterns in response to AG1 IA infection in resistant vs. susceptible rice genotypes. Unlike SAGE and microarray analysis or whole-genome sequencing analysis, this is the first study to generate extensive transcriptome data in rice after AG1 IA infection. Here, the comparison of RNA-seq data sets from resistant and susceptible genotypes revealed significant differences in expression among genes involved in defense signaling pathways and metabolic pathways and defined the characteristics of transcriptional regulation and identified key genes involved in rice sheath blight resistance.

\section{MATERIALS AND METHODS}

\section{Plant Growth Conditions and Pathogen Inoculation}

TeQing (a moderately resistant cultivar) and Lemont (a highly susceptible cultivar) seeds were obtained from the Rice Research Institute of Sichuan Agricultural University. The standard strain of $R$. solani, AG1 IA, was kindly provided by Pro. Er-Xun Zhou at South China Agricultural University and has been described previously (Shu et al., 2015). Fourth- and fifth-leaf-stage rice seedlings grown under natural light in a greenhouse (in which the temperature ranged from 20 to $30^{\circ} \mathrm{C}$ ) were inoculated with rice sheath blight fungus, AG1-IA. Before inoculation, the plants were transferred to a growth chamber for adaptive growth. Rice leaves were inoculated with potato dextrose agar (PDA) plugs containing mycelia (Jia et al., 2007). Agar blocks from a 2-day-old mycelia culture grown on PDA ( $5 \mathrm{~mm}$ in diameter to maintain uniform virulence) were placed on the abaxial surface of each leaf piece and covered with plastic wrap to maintain high humidity after inoculation (Supplementary Figure S1A). For the control group, plants were inoculated with agar blocks without fungus. The temperature ranged from 30 to $35^{\circ} \mathrm{C}$, the humidity inside the chamber was $>80 \%$, and the light was consistent throughout the process. Observation of the symptoms and trypan blue staining 
patterns was performed to determine the sampling time point (Marshall and Rush, 1980). Here, we chose five time points $(12,24,36,48$, and $72 \mathrm{~h})$ for RNA-seq.

At the time of harvest, for each treatment group including the control, leaflets were cut from each plant above the leaf sheath. Three biological replicates of lesions were collected randomly from each individual seedling. Leaves inoculated with agar blocks without fungus for $12 \mathrm{~h}$ served as the control for each cultivar. 36 samples [T12 (control), T12 h, T24 h, T36 h, T48 h, T72 h, L12 (control), L12 h, L24 h, L36 h, L48 h, and L72 h] were obtained for the present study. Samples for each treatment were harvested separately and immediately frozen in liquid nitrogen and stored at $-80^{\circ} \mathrm{C}$ until RNA extraction for transcriptome sequencing. All samples were sent to Beijing Biomarker Technologies, Inc., for transcriptome sequencing and cDNA library construction.

\section{Transcript Assembly, Sequence Alignment and Quantification}

To obtain expression profiles and predict gene structures, we mapped the pre-processed RNA-seq reads to each reference genome using the Cufflinks program ${ }^{1}$, TopHat $2^{2}$, and Bowtie ${ }^{3}$ (Li et al., 2009; Trapnell et al., 2010; Kim et al., 2013). We used the Nipponbare reference genome $\mathrm{MSU}^{4}$ as a reference sequence. When working with multiple RNA-seq samples, it is necessary to assemble the samples individually and then merge the resulting assemblies together. We used Cufflinks assemblies to reference annotation files and help separate new genes from known ones for use in downstream analyses. Then, the reads from each sample were mapped to the rice genome using TopHat version 2.1.1. The segments of the contiguously unmappable reads are then aligned against these synthetic sequences with Bowtie.

\section{Screening DEGs}

Differential expression was analyzed using the DESeq $\mathrm{R}$ package according to the package manual. Raw count data were prepared by a custom Perl script based on the results obtained from eXpress software and were imported into the DESeq framework. Experimental design information was also imported into the DESeq framework to form a count data set. The data were filtered to remove transcripts in the lowest $40 \%$ quantile of the overall sum of counts (irrespective of biological condition) to increase the rate of differentially expressed transcript detection. The estimate size factors function was used to estimate the effective library size to normalize the transcript counts. The estimate dispersions function was used to estimate dispersion. The nbinomTest function was used to determine whether there was differential expression between two conditions. False discovery rates (FDRs) were controlled using the Benjamini-Hochberg method at an FDR of 5\%. Transcripts per million (TPM) were used to measure the proportion of transcripts in the pool of RNA. Venn diagrams were used to analyze the overlap of differentially expressed transcripts among comparisons.

\footnotetext{
${ }^{1}$ http://cole-trapnell-lab.github.io/cufflinks/

${ }^{2} \mathrm{http}: / / \mathrm{ccb}$.jhu.edu/software/tophat/index.shtml

${ }^{3}$ http://bowtie-bio.sourceforge.net/

${ }^{4}$ http://rice.plantbiology.msu.edu/
}

\section{Pattern-Mining Based on Time Point and Gene Expression Pattern Analyses}

For a control and treatment time series, we designed a series of formulas containing the condition factor, the time factor, and the interaction between the two. Under this premise, we used the likelihood ratio test with a reduced model, which did not contain the interaction terms, to test whether the condition induces a change in gene transcription at any time point after the reference level time point (time $12 \mathrm{~h}$ ). From this analysis, a total of 4802 DEGs were identified between TeQing and Lemont leaves. We used self-organizing maps (SOMs) to analyze patterns in gene expression (Chang et al., 2010). A matrix of expression values for genes vs. samples was used as input data. Genes with similar expression were self-organized into nearby regions of a supra-hexagonal map. The resulting map was visualized to display the time-specific expression of the genes and was also further partitioned to obtain gene meta-clusters. For each metacluster, enrichment analysis was conducted using cluster Profiler packages. Following the analysis, transcripts of DEGs were used as the input for functional enrichment analysis.

\section{Functional Enrichment Analysis}

For functional gene annotation, the obtained unigene sequences were annotated by searching various databases, including the National Center for Biotechnology Information (NCBI), MSU Rice Genome Annotation Project ${ }^{5}$, Gene Ontology $(\mathrm{GO})^{6}$, the Kyoto Encyclopedia of Genes and Genomes (KEGG) ${ }^{7}$, and the China Rice Data Center 8 . Annotation information for homologous genes in these databases was used to represent the annotation of obtained unigenes. In addition, information regarding DEGs of the two rice varieties at different time points was collected from unigene annotations, and these genes were subjected to GO and KEGG significant enrichment analyses to identify the biological functions and related metabolic pathways in which these genes participate.

\section{Confirmation of the Infection-Responsive Expression Profiles by qRT-PCR}

To validate the Illumina sequencing data results, qRT-PCR analysis was performed on 36 RNA samples that were used in the preparation of sequencing libraries. Several genes that were co-expressed in both cultivars were analyzed by qRT-PCR at 12 , $24,36,48$, and $72 \mathrm{~h}$. Specific primers were designed according to individual gene sequences (Supplementary Table S1). cDNA was generated by reverse transcription using the Transcriptor First-Strand cDNA Synthesis Kit (Roche, Indianapolis, IN, United States). Reverse transcription was conducted using a stem-loop RT primer, ST-RT1, as described previously (Li et al., 2016). qRT-PCR experiments were performed on a Bio-Rad CFX96 Real-Time PCR System (Foster City, CA, United States)

\footnotetext{
${ }^{5}$ http://rice.plantbiology.msu.edu/

${ }^{6} \mathrm{http}: / / g e n e o n t o l o g y . o r g /$

${ }^{7}$ http://www.genome.jp/kegg/

${ }^{8}$ http://www.ricedata.cn
} 
in accordance with the manufacturer's instructions. cDNA $(2 \mu \mathrm{l})$ was amplified with $0.8 \mu \mathrm{l}$ of specific primers in a total reaction volume of $20 \mu \mathrm{l}$, and each reaction was performed four times. We used the UBQ gene as an internal control for data normalization. The $2^{\Delta \Delta C_{\mathrm{T}}}$ algorithm was used to calculate the gene expression levels.

\section{RESULTS}

\section{Early Symptoms of Rice Sheath Blight on Leaves}

The major symptoms observed in rice leaves during disease development after AG1 IA infection are shown in Supplementary Figure S1B. Both TeQing and Lemont leaves exhibited noticeable grayish spots approximately $36 \mathrm{~h}$ after inoculation. By direct observation, the spots on the Lemont leaves were bigger than those on the TeQing leaves. These symptoms were not apparent at $12 \mathrm{~h}$ or $24 \mathrm{~h}$ after inoculation on TeQing leaves but appeared on Lemont leaves at $24 \mathrm{~h}$. Trypan blue staining was performed on the leaf tissue near the inoculation site, and an ordinary optical microscope was used to observe hyphae growth. A small number of hyphae appeared at $12 \mathrm{~h}$ after inoculation in TeQing leaves, while Lemont leaves had more mycelial biomass than TeQing leaves at $12 \mathrm{~h}$. In TeQing leaves, mycelial biomass was increased at $24 \mathrm{~h}$, and hyphae were proliferative at $48 \mathrm{~h}$. In Lemont leaves, hyphae were denser at $24 \mathrm{~h}$ than in TeQing leaves, necrotic lesions and oval-shaped spots with yellow margins were found at $36 \mathrm{~h}$, and the lesion areas were larger and hyphae were more proliferative at $48 \mathrm{~h}$ (Supplementary Figure S2). Based on the symptoms observed on the leaves of the two cultivars, we chose $12,24,36,48$, and $72 \mathrm{~h}$ as time points for RNA-seq.

\section{RNA-seq Results of Transcriptome Samples}

To study the changes in gene expression in TeQing and Lemont leaves infected with AG1 IA at the initial infection stage as described above, we used next-generation sequencing to measure the transcription in rice leaves after AG1 IA inoculation. RNA sequencing data were generated from rice leaf samples at different time points. The transcriptome of each cultivar was analyzed at five time points with three biological replicates for each time point, and Illumina RNA-seq analysis of 36 samples yielded $246.30 \mathrm{Gbp}$ of data and 978,866,157 read pairs (obtained reads) (Supplementary Table S2A). After pre-processing of the reads, we obtained 2,357,278,781 single-end clean reads (total records) (Supplementary Table S2B). The total map ratio ranged from 73.78 to $93.30 \%$, and the unique map ratio ranged from 44.93 to $72.25 \%$. However, many reads could not be mapped to the reference genomes (6.7-26.22\%) (Supplementary Table S2B). As these datasets were generated from different rice cultivars and at different time points, expression levels in all samples were calculated uniformly, as described previously (Wang et al., 2014). We used sample-to-sample correlation analysis for the data exploration analysis. The overall relatedness of the transcriptome at different times was determined by a cluster dendrogram generated for the TeQing and Lemont samples (Supplementary Figure S3). For all samples, the three biological replicates (TeQing and Lemont) showed good correlation, and the transcriptome data were closely related at each time point. Also PCA was used to assess the similarity between samples across conditions using separate samples from the different biological conditions according to the first principal component (PC1) and the second principal component (PC2), meaning that the biological variability between two groups was the main source of variance in the data (Supplementary Figure S4). This may occur for several reasons, such as RNA contamination between samples (even between biological replicates) or slight differences between library concentrations, since they may be difficult to distinguish with high precision.

\section{Differential Gene Analysis of TeQing and Lemont Leaves at Different Time Points}

To determine which genes exhibit expression changes and at what stage these changes occur, comparisons between the rice cultivars, with controls, were performed. A comparison between the two varieties yielded data regarding differentially expressed rice genes at different time points after AG1 IA inoculation (Table 1). After AG1 IA inoculation, the number of up-regulated genes exceeded the number of down-regulated genes, and genes were more up-regulated in Lemont leaves than in TeQing leaves at all time points. At 12 h, 4,205 DEGs (2,348 up-regulated, 1,857 down-regulated) were identified in Lemont leaves, compared with 1,676 DEGs in TeQing leaves (1,021 up-regulated, 655 down-regulated), suggesting that Lemont leaves are more susceptible to AG1 IA than TeQing leaves and that the infection pressure is greater on Lemont plants than on TeQing plants. The number of DEGs in Lemont leaves was highest at $24 \mathrm{~h}$ (7,041 DEGs; 4,781 up-regulated, 2260 downregulated) and $48 \mathrm{~h}$ (5,920 DEGs; 4,201 up-regulated, 1,719

TABLE 1 | Statistics of differentially expressed genes.

\begin{tabular}{lcccc}
\hline Combination & $\begin{array}{c}\text { DEG set } \\
\text { name }\end{array}$ & $\begin{array}{c}\text { Up- } \\
\text { regulated }\end{array}$ & $\begin{array}{c}\text { Down- } \\
\text { regulated }\end{array}$ & $\begin{array}{c}\text { All } \\
\text { DEGs }\end{array}$ \\
\hline TCK_vs_T12 & T12 & 1021 & 655 & 1676 \\
TCK_vs_T24 & T24 & 3957 & 1350 & 5307 \\
TCK_vs_T36 & T36 & 789 & 692 & 1481 \\
TCK_vs_T48 & T48 & 2473 & 650 & 3123 \\
TCK_vs_T72 & T72 & 1095 & 211 & 1306 \\
LCK_vs_L12 & L12 & 2348 & 1857 & 4205 \\
LCK_vs_L24 & L24 & 4781 & 2260 & 7041 \\
LCK_vs_L36 & L36 & 2025 & 1034 & 3059 \\
LCK_vs_L48 & L48 & 4201 & 1719 & 5920 \\
LCK_vs_L72 & L72 & 1543 & 483 & 2026
\end{tabular}

TCK_vs_T12, TCK_vs_T24, TCK_vs_T36, TCK_vs_T48, and TCK_vs_T72 represent the comparison of TeQing leaves infected with AG1 IA for 12, 24, 36, 48, or $72 h$ and TeQing control leaves after $12 h$, respectively; the same follows for LCK_vs_L12, LCK_vs_L24, LCK_vs_L36, LCK_vs_L48, and LCK_vs_L72. Unigenes with a false discovery rate (FDR) no greater than 0.01 and reads per kb per million reads (RPKM) between samples of no less than 2 [fold change (FD) $\geq 2$ ] were considered differentially expressed genes (DEGs). 
down-regulated) after AG1 IA inoculation and exceeded the number of DEGs in TeQing leaves at the same time points. The data suggest that after AG1 IA infection, differential gene expression was induced to a greater extent in the susceptible cultivar (24 and $48 \mathrm{~h}$ ) than in the resistant cultivar. As for the differences observed at 24 and $48 \mathrm{~h}$ compared with other time points, we speculate that primary pathogen colonization occurred within $24 \mathrm{~h}$ based on the appearance of symptoms and on hyphae growth observed under a microscope. The pathogen then established re-infection, causing the degree of infection to be enhanced at $48 \mathrm{~h}$.

We also analyzed both cultivar controls and samples at 12 , 24, 36, 48, and $72 \mathrm{~h}$ after AG1 IA inoculation. The samples were subjected to multi-group differential analysis to identify DEGs in rice associated with response to AG1 IA infection. DEG sets at the same time points in both cultivars (L12-T12, L24-T24, L36-T36, L48-T48, and L72-T72) were compared (Figure 1). At 24 and $48 \mathrm{~h}$ after AG1 IA inoculation, 3,490 and 2,171 DEGs were identified in both cultivars, which was more than at other time points. In addition, the time-dependent expression of DEGs was analyzed by comparing the expression at different time points in each cultivar (T12-T24-T36-T48-T72 h or L12-L24-L36-L48-L72 h) (Figure 2). In TeQing leaves, 82 common DEGs exhibited sustained expression at 12, 24, 36, 48, and $72 \mathrm{~h}$ after inoculation (51 up-regulated; 31 down-regulated). At 12 h, 878 specific DEGs were identified (652 up-regulated; 323 down-regulated). At 24 h, 2,802 specific DEGs were identified (2,098 up-regulated; 762 down-regulated), and 608 specific DEGs were identified (459 up-regulated; 163 down-regulated) at $48 \mathrm{~h}$. In Lemont leaves, 241 genes had sustained expression at 12, 24, 36,48 , and $72 \mathrm{~h}$ after inoculation (158 up-regulated; 73 downregulated). At 12 h, 1,841 specific DEGs were identified (1,488 up-regulated; 865 down-regulated). At 24 h, 2,770 specific DEGs were identified (2,054 up-regulated; 949 down-regulated). At 48 h, 1,490 specific DEGs were identified (1,088 up-regulated; 590 down-regulated). Overall, from 12 to $72 \mathrm{~h}$ after AG1 IA infection, more continuously expressed genes were observed in Lemont leaves than in TeQing leaves.

To identify the functional distribution of unigenes, we used GO annotations to classify the enriched DEGs between the control and AG1 IA-treated groups. These genes were involved in all aspects of biological activity, and intriguingly, the distribution of annotated unigenes in the two cultivars showed highly similar patterns (Supplementary Figure S5). All the unigenes annotated in the GO database were classified into three main categories. Within biological processes, "photosynthesis," "generation of precursor metabolites and energy," "growth," and "cell death" were the four most common GO terms. For the cellular components category, most of the unigenes were related to "peroxisome," "nucleolus," and "Golgi apparatus." In the molecular functions category, unigenes were mostly involved in "binding," followed by "nuclease activity" and "enzyme regulator activity." These highly enriched GO terms mainly refer to the maintenance of the basic regulation and metabolic functions of rice after AG1 IA infection. Additionally, GO terms related to special disease resistance functions, such as "cell death" in the biological process category and "oxygen binding" in the molecular function category, were found. From the results, we selected GO terms related to pathogen resistance to analyze the differences between TeQing and Lemont leaves. For example, genes related to photosynthesis were maintained at a relatively stable level in TeQing leaves compared with Lemont leaves in the early stage of infection. Genes related to the generation of precursor metabolites and energy exhibited a larger increase in expression in TeQing leaves than in Lemont leaves at $24 \mathrm{~h}$. For genes annotated by the GO terms "cell death," "peroxisome," and "oxygen binding," we also found that the change in expression was larger in TeQing leaves than in Lemont leaves. From the GO terms, we obtained an overview of ontology content and revealed the global biological activities occurring in the TeQing and Lemont leaves after AG1 IA infection. Our results suggest that at early time points in the rice-AG1 IA interaction, plant defense-related genes are more active in Lemont leaves than in TeQing leaves. Thus the resistant and susceptible cultivars had specific reactions in response to AG1 IA infection.

\section{Analysis of the Dynamic Gene Expression Patterns of Both Rice Cultivars}

Self-organizing maps were used to identify the dynamic changes among the 4,802 common DEGs at different time points to compare the two rice varieties (Supplementary Table S3), and multi-group expression pattern clustering analysis was performed. Each component plane shows the hexagonal variation in gene expression [log2 (pond/upland)] at one time point, using a color gradient from blue to red to indicate up- and downregulation (see the colored bar). Gene sets generated using the SOM algorithm were projected to different small grids, and adjacent gene grids have similar expression patterns (Figure 3A). The lattices are clustered according to gene expression levels, and lattices belonging to the same cluster have the same color (Figures 3B,C). Finally, the characteristic values of the gene expression levels in each lattice were extracted and are presented in Figures 4, 5 to show the expression levels of the lattice genes. GO classification analysis was performed to determine whether the differentially regulated genes in each cluster were significantly associated with a specific biological process, molecular function or cellular component (Supplementary Tables S4, S5). From the SOM, we found that both the TeQing and Lemont DEGs were primarily concentrated during the early stage of infection (12 and $24 \mathrm{~h}$ ). The two rice cultivars exhibited very different defense patterns after AG1 IA infection. We selected several clusters to further analyze the differential expression patterns in the two rice cultivars.

In the patterns of cluster No. 17 in TeQing and cluster No. 15 in Lemont, the levels of DEGs exhibited sustained induction after AG1 IA inoculation. The up-regulation of these genes began within $12 \mathrm{~h}$ and persisted throughout the $72 \mathrm{~h}$ experimental period. The sustained accumulation of this gene type suggests important biological significance in response to AG1 IA. We collected GO annotations to classify the DEGs identified in TeQing and Lemont leaves. Cluster 17 in TeQing was 

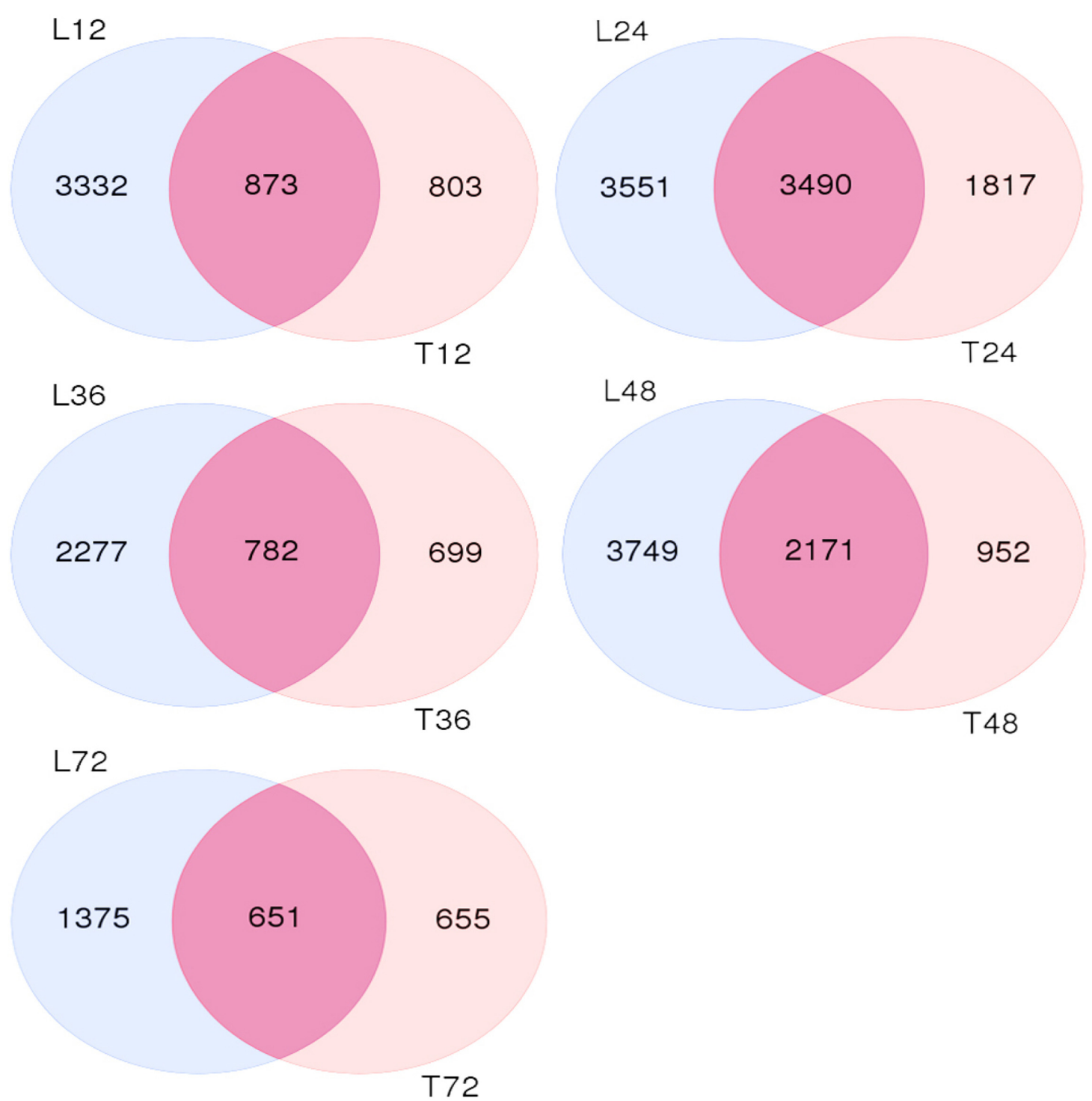

FIGURE 1 | Venn diagram of differentially expressed genes found in both rice cultivars after AG1 IA infection at the same time points. L12, L24, L36, L48, and L72 denote differentially expressed gene sets obtained from Lemont leaves at 12, 24, 36, 48, and 72 h after AG1 IA infection, respectively, compared to control leaves at $12 \mathrm{~h}$; the same is shown for T12, T24, T36, T48, and T72.

enriched in genes involved in cell death, responses to extracellular stimuli, peroxisomes, the Golgi apparatus, and enzyme regulation and signal transduction activities. Cluster No. 15 in Lemont mainly included genes involved in the generation of precursor metabolites and energy, the regulation of cell death, the Golgi apparatus, lipid binding and motor activity.

In the patterns of cluster No. 11 in TeQing and cluster No. 6 in Lemont, we found that the level of induction of the DEGs decreased steadily relative to the original level and reached a minimum after $72 \mathrm{~h}$ of plant-pathogen interaction. The gene expression levels in cluster No. 11 in TeQing showed a steeper decline than those in cluster No. 6 in Lemont. GO analysis showed that cluster No. 11 in TeQing was enriched in genes related to the nucleolus and that cluster No. 6 in Lemont was mainly enriched in genes involved in the generation of precursor metabolites and energy, cell death, photosynthesis, cellular homeostasis, nucleolus, oxygen binding and nuclease activity.

In the patterns of cluster No. 12 and cluster No. 19 in TeQing, the levels of DEGs decreased at $12 \mathrm{~h}$ and then peaked at $24 \mathrm{~h}$ in cluster No. 12. Cluster No. 19 showed an opposite expression pattern: the levels were high at $12 \mathrm{~h}$ but decreased at $24 \mathrm{~h}$. Based on the GO classifications, we found that in the two dynamic expression patterns, cluster No. 12 was enriched in genes involved in the generation of precursor metabolites, cellular homeostasis, nucleolus, and enzyme regulator activity, and cluster No. 19 was mainly enriched in genes related to the regulation of gene expression, epigenetics, the cell cycle, 

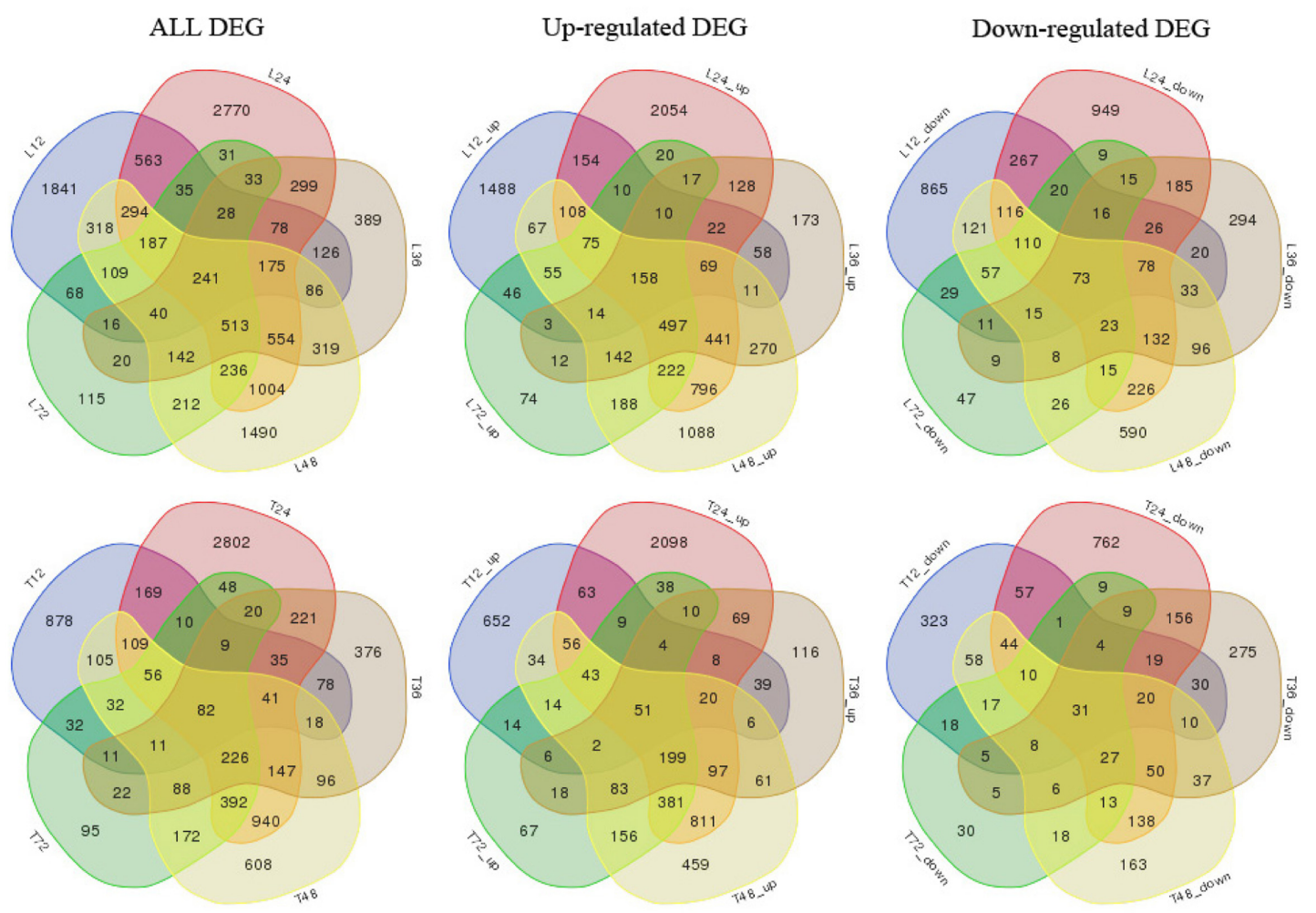

FIGURE 2 | Venn diagram of differentially expressed genes in both rice cultivars at different time points after AG1 IA inoculation. T12, T24, T36, T48, and T72 denote differentially expressed gene sets obtained by comparing TeQing samples at 12, 24, 36, 48, and $72 \mathrm{~h}$ after AG1 IA inoculation, respectively, with the control sample at 12 h; L12, L24, L36, L48, and L72 denote differentially expressed gene sets obtained by comparing Lemont samples at 12, 24, 36, 48, and 72 h after AG1 IA inoculation, respectively, with the control sample at $12 \mathrm{~h}$. All DEGs, all differentially expressed genes; Up-regulated DEGs, up-regulated genes; Down-regulated DEGs, down-regulated genes.

nucleoplasm, Golgi apparatus, cytoskeleton, nucleolus, signal transduction activity and oxygen binding.

Interestingly, the genes of Cluster No. 20 and No. 21 in the moderately resistant cultivar TeQing were continuously upregulated after AG1 IA inoculation. The expression levels of these genes peaked at $72 \mathrm{~h}$ after infection treatment, and these genes were involved in cell death, responses to extracellular stimuli, cell communication, cellular homeostasis, the cytoskeleton and oxygen binding. In Cluster No. 10 in Lemont, the DEGs upregulated at $36 \mathrm{~h}$ were analyzed using GO enrichment. This cluster was significantly enriched in genes associated with the generation of precursor metabolites and energy, cellular homeostasis, cell death, the Golgi apparatus and oxygen binding.

\section{Analysis of Metabolic Pathways after AG1 IA Inoculation}

To further investigate the biological functions and interactions of differentially regulated transcripts specific to TeQing and Lemont leaves after AG1 IA inoculation, pathway-based analysis was conducted using KEGG. To further analyze the differences between the two rice cultivars, we examined the KEGG categories with p.adjust $<0.01$ that were used to classify up-regulated genes into different biological processes. These categories are presented in Supplementary Tables S6, S7, and the unigenes involved in resistance-associated metabolic pathways were included.

At $12 \mathrm{~h}$, the highly represented pathways in TeQing leaves compared with Lemont leaves were glycine, serine and threonine metabolism ( .adjust $=2.72 \mathrm{E}-05$ ), phenylalanine, tyrosine and tryptophan biosynthesis ( .adjust $=2.72 \mathrm{E}-05$ ) and phenylpropanoid biosynthesis (p.adjust $=0.001256$ ). At $24 \mathrm{~h}$, the primary pathways enriched in TeQing leaves were phenylalanine, tyrosine and tryptophan biosynthesis (p.adjust $=8.54 \mathrm{E}-10)$, alpha-linolenic acid metabolism $(p$.adjust $=2.25 \mathrm{E}-08)$, phagosome $(p$.adjust $=6.37 \mathrm{E}-08)$, and plant-pathogen interaction ( $p$.adjust $=1.66 \mathrm{E}-06$ ). In Lemont leaves, the phenylalanine, tyrosine and tryptophan biosynthesis (p.adjust $=3.67 \mathrm{E}-10)$ and plant-pathogen interaction (p.adjust $=1.77 \mathrm{E}-09)$ pathways were significantly enriched. In addition, we found that the valine, leucine and isoleucine degradation ( $p$.adjust $=0.003265$ ), phenylalanine metabolism (p.adjust $=0.00423$ ) and phenylpropanoid biosynthesis ( $p$.adjust $=0.000247$ ) pathways were also enriched in TeQing leaves at $24 \mathrm{~h}$.

To further analyze the effects of related metabolic pathways on plant resistance, TPM was used to compare the expression of key genes involved in metabolic pathways between TeQing 


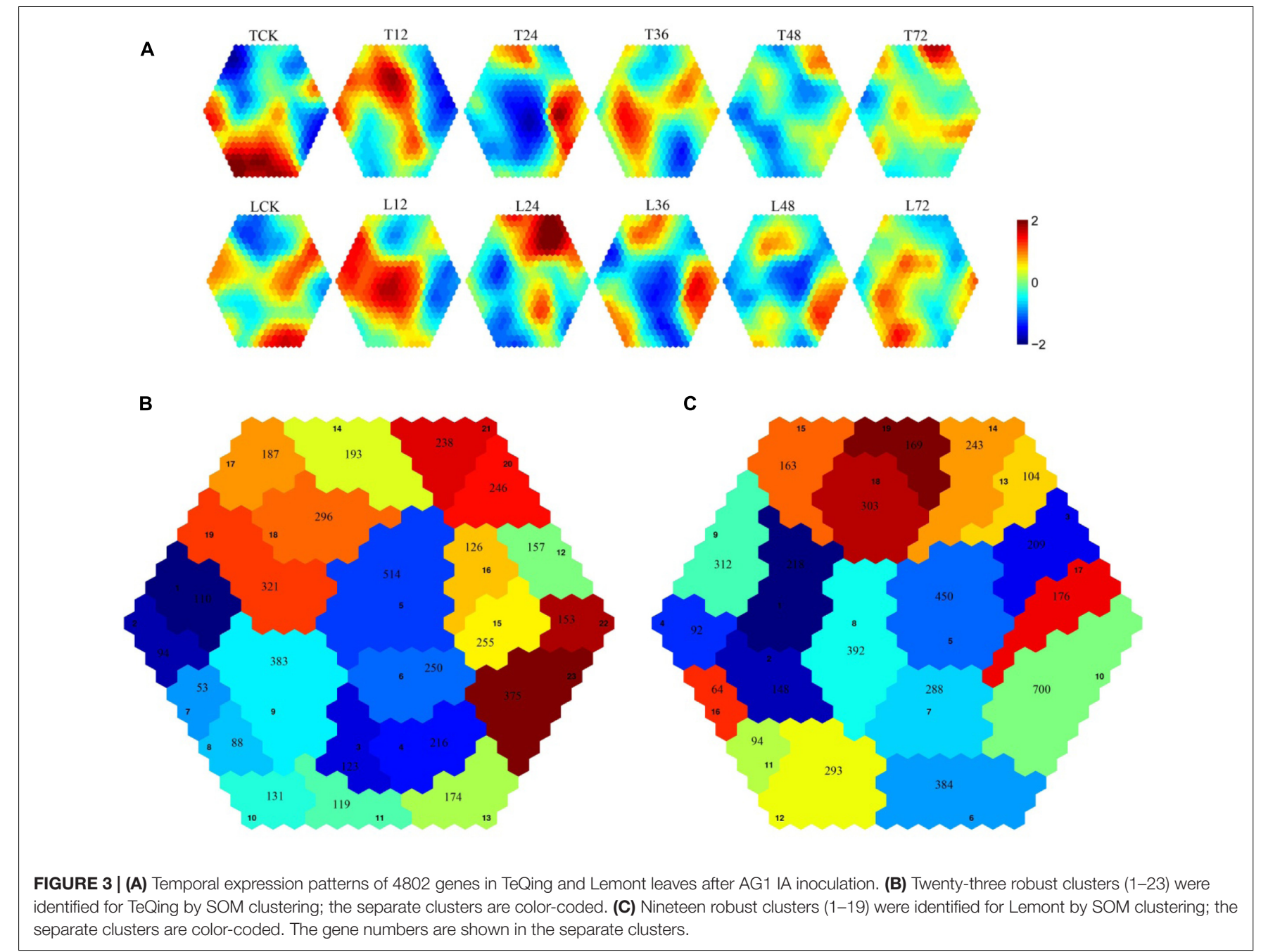

and Lemont leaves (Figure 6). In addition to photosynthesis metabolism, PsbS was rapidly increased in TeQing leaves after AG1 IA treatment. Additionally, we found that OsLFNR1 and OsLFNR2 were both increased at $12 \mathrm{~h}$ in the two rice varieties. OsLFNR1 was more highly expressed in TeQing leaves than in Lemont leaves. Based on the finding that glycine, serine and threonine metabolism ( $p$.adjust $=2.72 \mathrm{E}-05)$ and glyoxylate and dicarboxylate metabolism ( $p$.adjust $=2.72 \mathrm{E}-05$ ) were induced in TeQing leaves at $12 \mathrm{~h}$, we examined the expression of genes that were closely related to photorespiration. GLO1 and GLO4 were both increased in TeQing and Lemont leaves, and GLO4 was more highly expressed in TeQing leaves at $12 \mathrm{~h}$. In addition, other genes, such as serine hydroxymethyl transferase (OsSHM1), catalase (OsCATC), glutamine synthetase (OsGS2) and ribulose-1,5-bisphosphate carboxylase/oxygenase small subunit (OsRBCS2), that participate photorespiration were found to be increased at $12 \mathrm{~h}$. Based on the observation that plant-pathogen interaction pathways were induced at $24 \mathrm{~h}$, we found that rice LysM receptor-like kinase (OsCERK1), rice chitin receptor chitin oligosaccharide elicitor-binding protein (OsCEBiP) and OsFLS2 were expressed in TeQing and Lemont leaves. These results were corroborated by the fact that the expression of CEBiP and CERK1 was maximal at $24 \mathrm{~h}$. Most of the genes related to calcium signaling were down-regulated at $12 \mathrm{~h}$ but increased at $24 \mathrm{~h}$. Additionally, mitogen-activated protein kinase (MAPK), WRKY, and pathogenesis-related genes as well as other genes were found to be involved in this metabolic pathway.

To identify potential regulatory genes tightly related to phenylalanine metabolism at $12 \mathrm{~h}$, we identified DEGs by comparing the expression changes between the two varieties. Of the genes involved in phenylalanine metabolism that were expressed at $12 \mathrm{~h}$, more were expressed at a higher level in TeQing leaves than in Lemont leaves; the difference between the two varieties can be seen in the heatmap (Figure 7). Genes associated with the KEGG term alpha-linolenic acid metabolism, which is an important precursor of jasmonic acid (JA), were found in TeQing and Lemont leaves. Corresponding genes were identified, and their expression is shown in Figure 8. These genes included 12-oxophytodienoic acid reductase, 3-ketoacyl-CoA thiolase, lipoxygenase, allene oxide cyclase, phospholipase, and cytochrome P450, which all participate in JA biosynthesis (Wang 


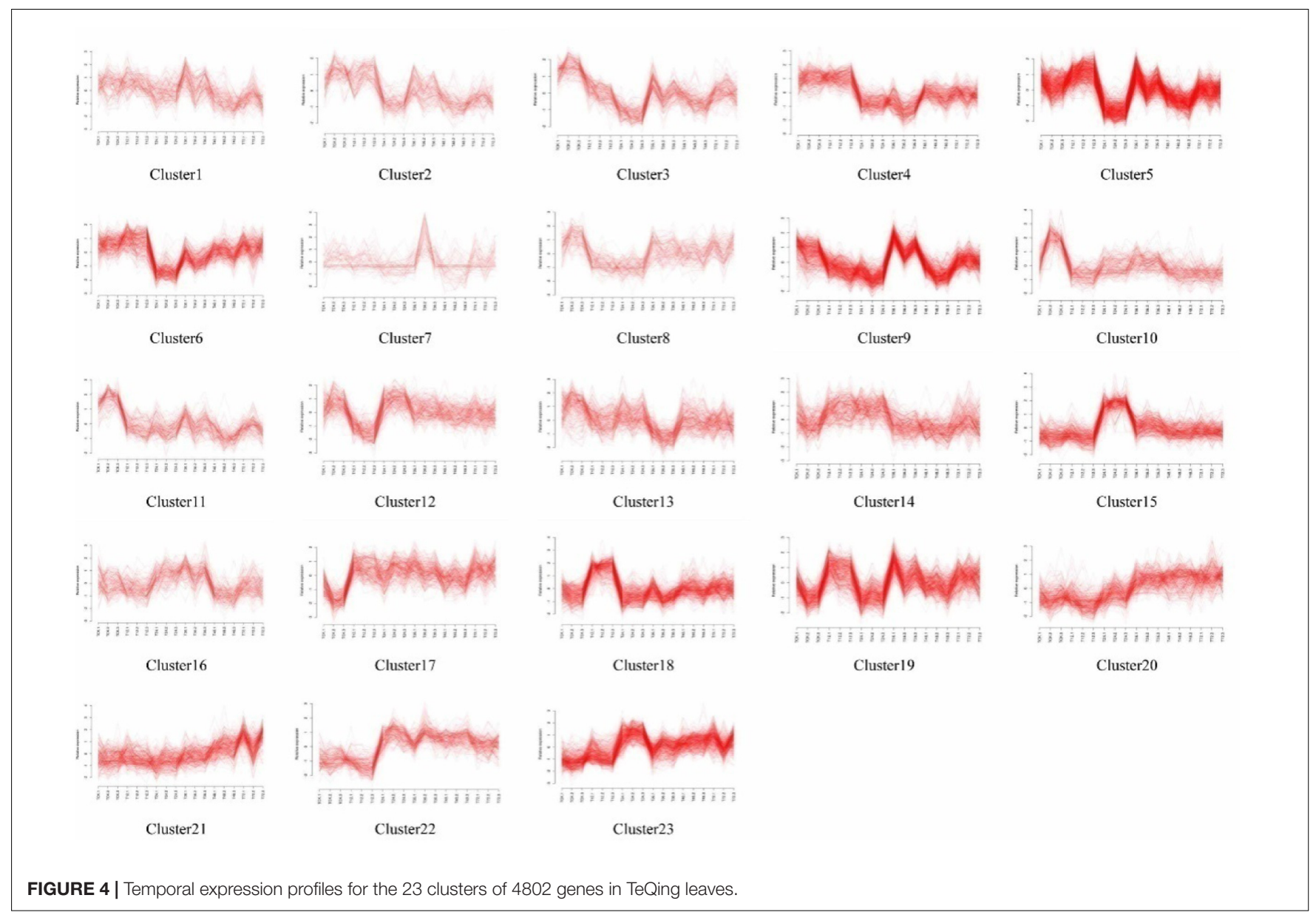

et al., 2000; Zhang et al., 2005; Schaller and Stintzi, 2009). Because more than $90 \%$ of salicylic acid (SA) is synthesized via the isochorismate synthase (ICS) pathway (Wildermuth et al., 2001), we used OsICS1 to analyze the changes in SA synthesis in the two cultivars. OsICS1 was expressed in both TeQing and Lemont leaves, exhibiting maximum expression at $12 \mathrm{~h}$ and then decreasing. However, TeQing leaves expressed more OsICS1 than Lemont leaves at $12 \mathrm{~h}$ (Figure 6).

Our results revealed that the metabolic pathways of both varieties participate in similar mechanisms after AG1 IA infection, but in the initial period of pathogen infection, TeQing leaves exhibited an earlier response to pathogen infection than Lemont leaves. However, during pathogen infection, genes involved in alpha-linolenic acid metabolism were upregulated in Lemont leaves after $24 \mathrm{~h}$ compared with TeQing leaves, and related resistance-associated metabolic pathways were significantly enriched in Lemont leaves. Comparing the expression of genes involved in phenylalanine metabolism and alpha-linolenic acid metabolism in TeQing and Lemont leaves indicated a time-shift in the expression patterns. Further research into DEGs involved in metabolic pathways is needed to understand the mechanisms underlying AG1 IA resistance.

\section{Quantitative RT-PCR (qRT-PCR) Validation of DEGs}

The RNA-seq data were validated by quantitative reverse transcription PCR (qRT-PCR) analysis. The rice pathogenesisrelated proteins PR1, PR5, and PR10 were selected for validation (Maruthasalam et al., 2007; Wu et al., 2011), as was WRKY72, a member of the WRKY gene superfamily in rice whose expression is reportedly induced expression during the infection of Magnaporthe oryzae, Ustilaginoidea virens and X. oryzae pv. oryzicola (Chao et al., 2014). In addition, several genes involved in metabolic pathways were also selected for validation. The Ct values obtained were normalized to those of the internal control, and the fold change upon AG1 IA infection was calculated for both rice cultivars. In addition, the fold change of transcripts in infected TeQing and Lemont leaves with respect to control conditions were also calculated. The differential expression values of all selected transcripts obtained using qRT-PCR analysis were plotted along with the RNA-seq data and their patterns were compared (Supplementary Figure S6). The qRT-PCR analyses showed trends in expression that were consistent with those found by RNA-seq, suggesting that the Illumina data are relatively reliable. 


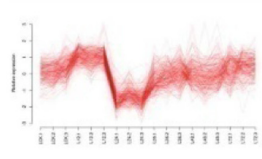

Cluster1

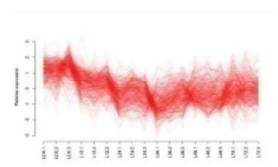

Cluster6

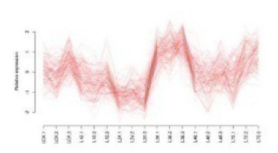

Cluster11

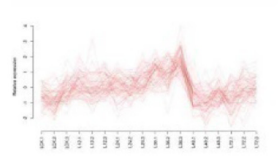

Cluster16

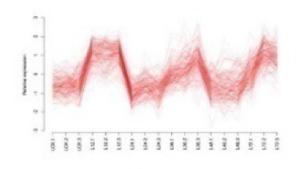

Cluster2

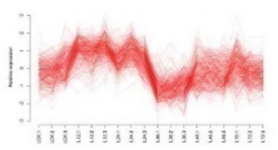

Cluster7

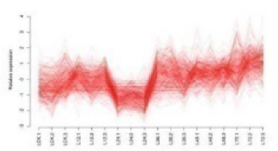

Cluster12

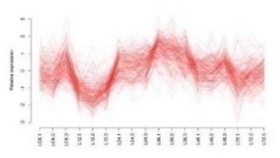

Cluster17

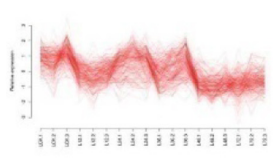

Cluster3

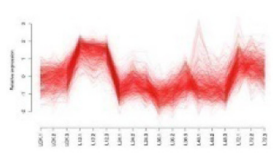

Cluster8

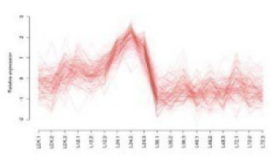

Cluster13

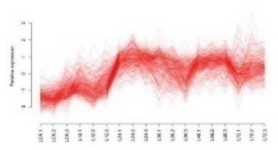

Cluster18

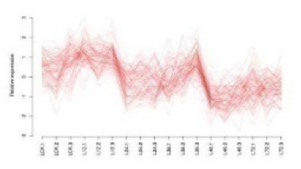

Cluster4

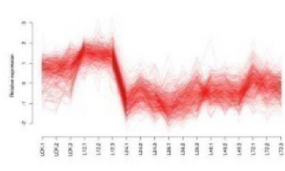

Cluster9

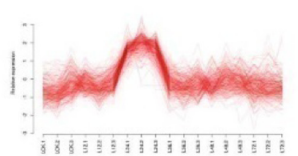

Cluster14

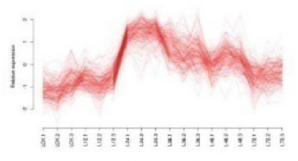

Cluster19
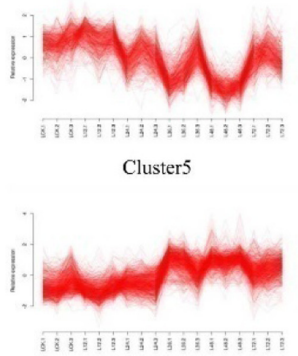

Cluster10

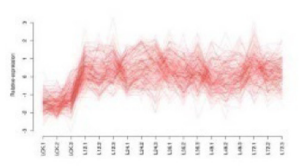

Cluster15

FIGURE 5 | Temporal expression profiles for the 19 clusters of 4802 genes in Lemont leaves.

\section{DISCUSSION}

In recent years, RNA-seq has been widely used in the screening of different genes, functional gene mining and the analysis of molecular mechanisms of disease resistance in plant-pathogen interactions (Kawahara et al., 2012). It was previously reported that differential expression analysis at the isoform level can provide a more comprehensive overview of gene regulation than gene-level expression patterns (Nakashima and YamaguchiShinozaki, 2009). Here, using two different types of rice cultivars, we revealed that the differential regulation of gene expression exhibited a very different defense pattern between cultivars and that TeQing leaves respond more quickly during the initial infection stage. These data show that in both TeQing and Lemont leaves, DEGs were primarily concentrated in the early stages of infection. Our results may facilitate the discovery and annotation of important genes in the defense response, physiology and metabolism of plants and provide comprehensive infectionresponsive expression profiles for the rice response to AG1 IA. Further investigation of these differentially regulated genes will provide insights into the differential pathogen responses of different rice cultivars.

\section{Gene Expression Changes in Response to AG1 IA Infection}

Previous studies have shown that in susceptible plants, a slow response and weaker defense signals allow pathogens to travel through the tissue and damage the plant. Such molecular changes affect later alterations in plant appearance, physiology and biochemistry (Que et al., 2014). Research has shown that the resistance of a plant to necrotrophic fungi is based on its ability to counteract the toxic effects of reactive oxygen species (ROS), which may involve the production of antimicrobial metabolites, such as phytoalexins, and the induction phytohormone-regulated signaling pathways, predominantly the JA and cell death pathways (Glazebrook, 2005). Various numbers of GO terms were enriched in each rice cultivar after AG1 IA infection. The major differences between TeQing and Lemont plants were observed in the timing of host recognition and in the speed and effectiveness of the defense response to AG1 IA invasion. In addition, the number of genes expressed in Lemont leaves was greater than that in TeQing leaves throughout AG1 IA infection, which may indicate that the different rice cultivars activate different mechanisms upon AG1 IA infection. It has been suggested that during plant-pathogen interactions, the role of primary metabolism is to support the cellular energy requirements for plant defense responses to establish a favorable energy balance for defense (Kangasjärvi et al., 2012; Andolfo and Ercolano, 2015). Through various metabolic processes, plants produce many metabolites that could improve disease resistance, and these metabolites form an important material basis for plant resistance systems (Rojas et al., 2014). Here, based on our KEGG analysis, we compiled evidence from the literature to show that upon exposure to pathogens, plants induce genes associated with primary metabolic pathways. Transcriptome sequencing analysis showed that most of the genes and pathways induced in both cultivars were similar. This is consistent with previous data suggesting that after pathogens infect plants, many metabolic pathways are affected, and gene expression in the transcription network is disturbed (Bolton et al., 2008; Coram et al., 2008). 

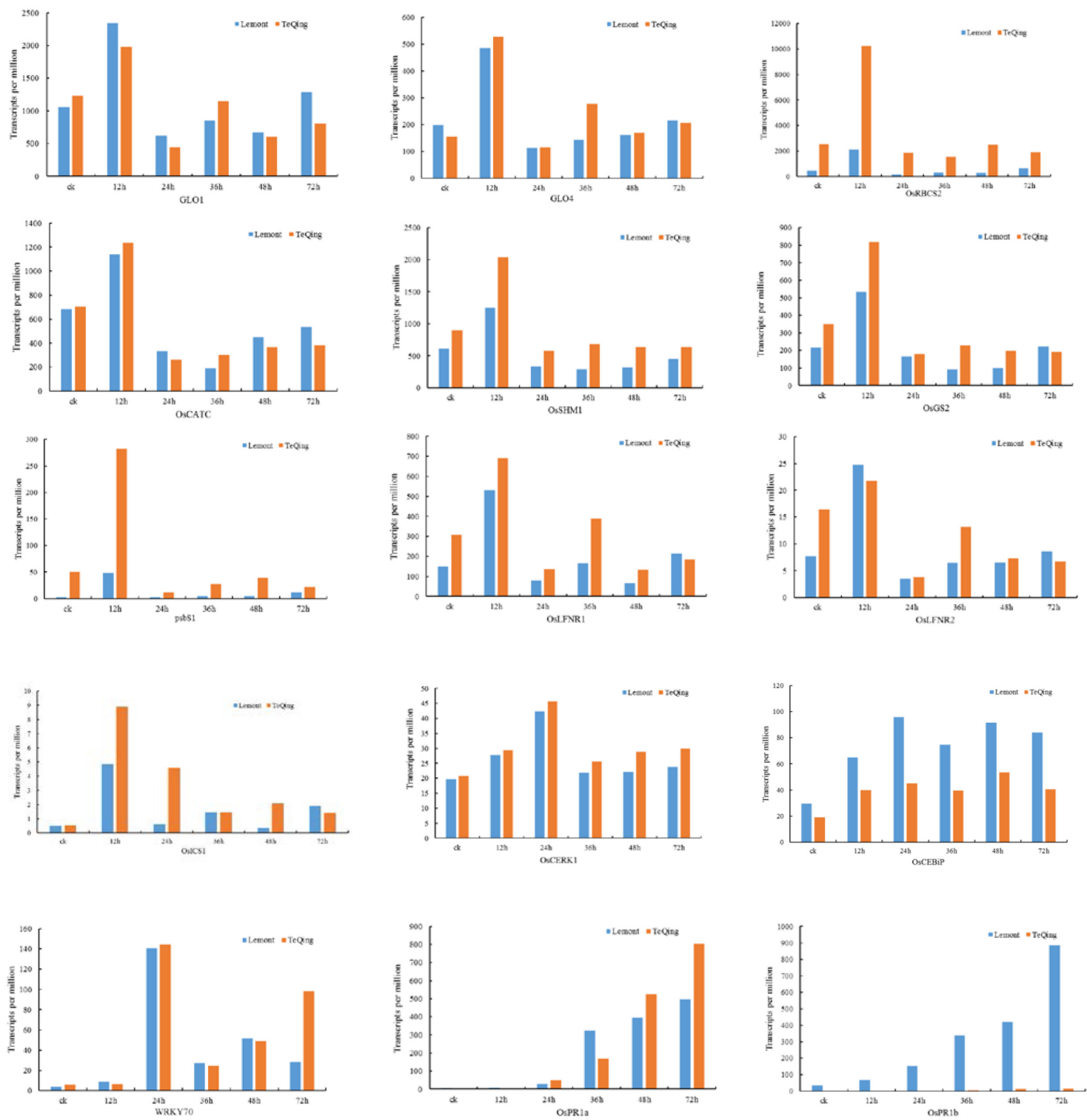

FIGURE 6 | Expression of genes involved in photosynthesis, photorespiration and other processes in TeQing and Lemont leaves based on transcripts per million (TPM).

\section{Photosynthesis and Photorespiration}

The defense response requires energy, and this causes an increased demand for photosynthesis, as photosynthesis provides a carbon source for the synthesis of defense compounds (Hammerschmidt, 1999). Previous studies have revealed the link between the defense response and photosynthesis (Kerchev et al., 2012). Non-photochemical quenching (NPQ) is an essential process that protects plants from excess light energy and resulting damage from ROS formation (Müller et al., 2001). Göhre et al. (2012) found that activation of defense mechanisms by pathogen-associated molecular patterns (PAMPs) could lead to a rapid decrease in NPQ and that NPQ also influences several PAMP-triggered immune response processes. In addition, they proposed that NPQ might play a role in positively regulating PAMP-triggered immunity (PTI). PsbS is a central player involved in NPQ and controls the macro-organization of photosystem II complexes (Niyogi et al., 2005; Kereiche et al., 2009). The expression of PsbS observed here suggests that NPQ might be increased upon AG1 IA infection. The ferredoxinthioredoxin system is a well-known mechanism that activates light-driven metabolic reactions in chloroplasts. Light-driven redox chemistry also provides plants with a mechanism for generation of ROS, which are key players in the relay of stress signals in photosynthetic tissues (Buchanan and Balmer, 2005). A previous study showed that transient increases in the levels of ROS in chloroplasts have vital signaling roles in the initiation of 


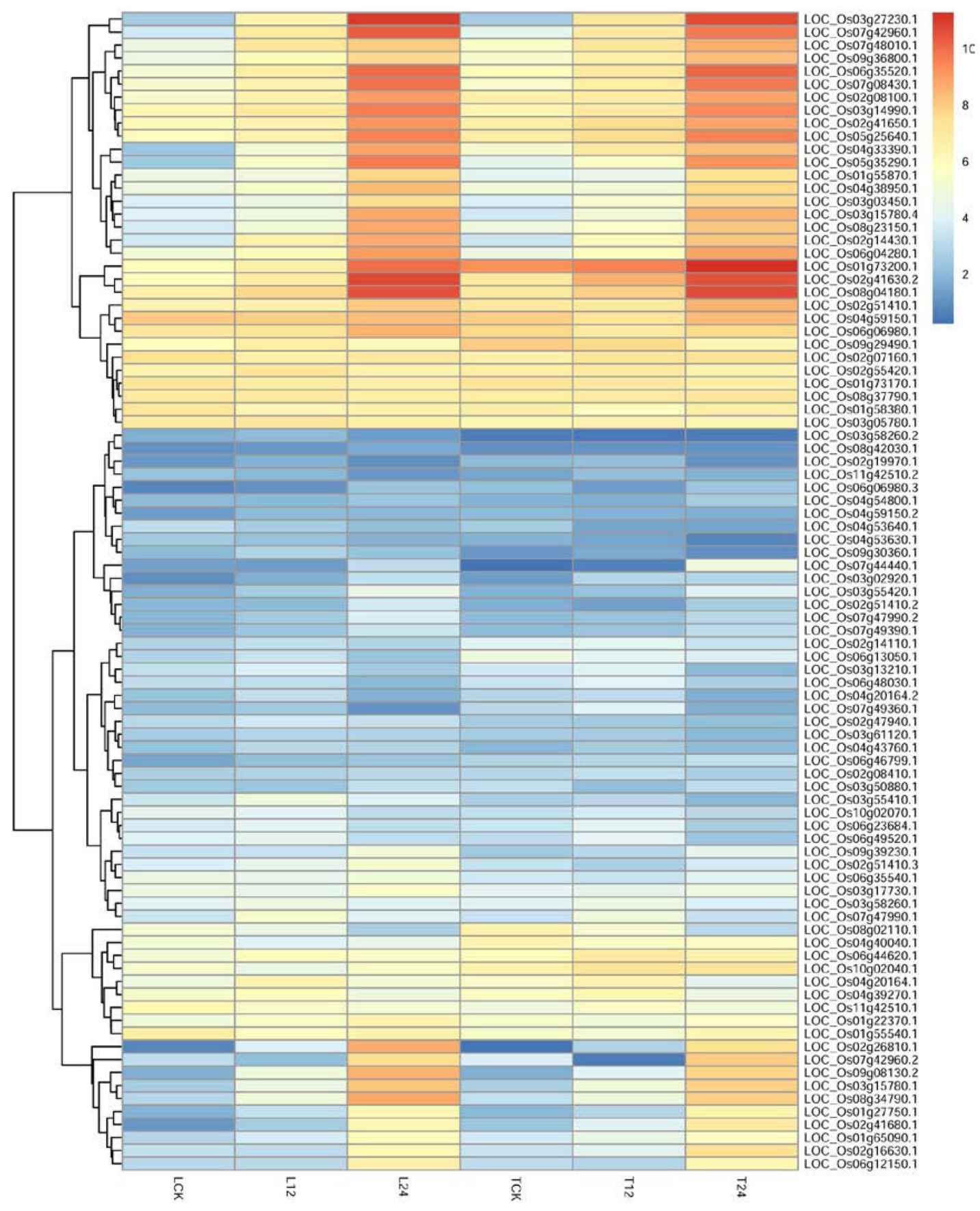

FIGURE 7 | Detailed expression profiles of genes related to phenylalanine metabolism pathways at 12 and $24 \mathrm{~h}$.

immune reactions upon attempted infection in different cell types (Gechev et al., 2006). In addition to ROS formation, nitric oxide (NO) generation and the induction of SA and JA biosynthesis occur in chloroplasts and contribute to the specificity of immune reactions (Boller and He, 2009). Here, we found that genes related to photosynthesis were increased at $12 \mathrm{~h}$ and then decreased. As previous study suggested that the decrease in photosynthesis after infection might be an active mechanism of the plant defense program that limits the carbon source availability for the pathogen or that it may result from the prioritizing of metabolic processes that contribute to plant defense responses (Bolton, 2009).

Photorespiration, an important process that produced $\mathrm{H}_{2} \mathrm{O}_{2}$, is critical in the plant defense response and regulates the cell redox state. Research has shown that photorespiration reduces the Mehler reaction rate, mitigates the potential hazards of ROS 


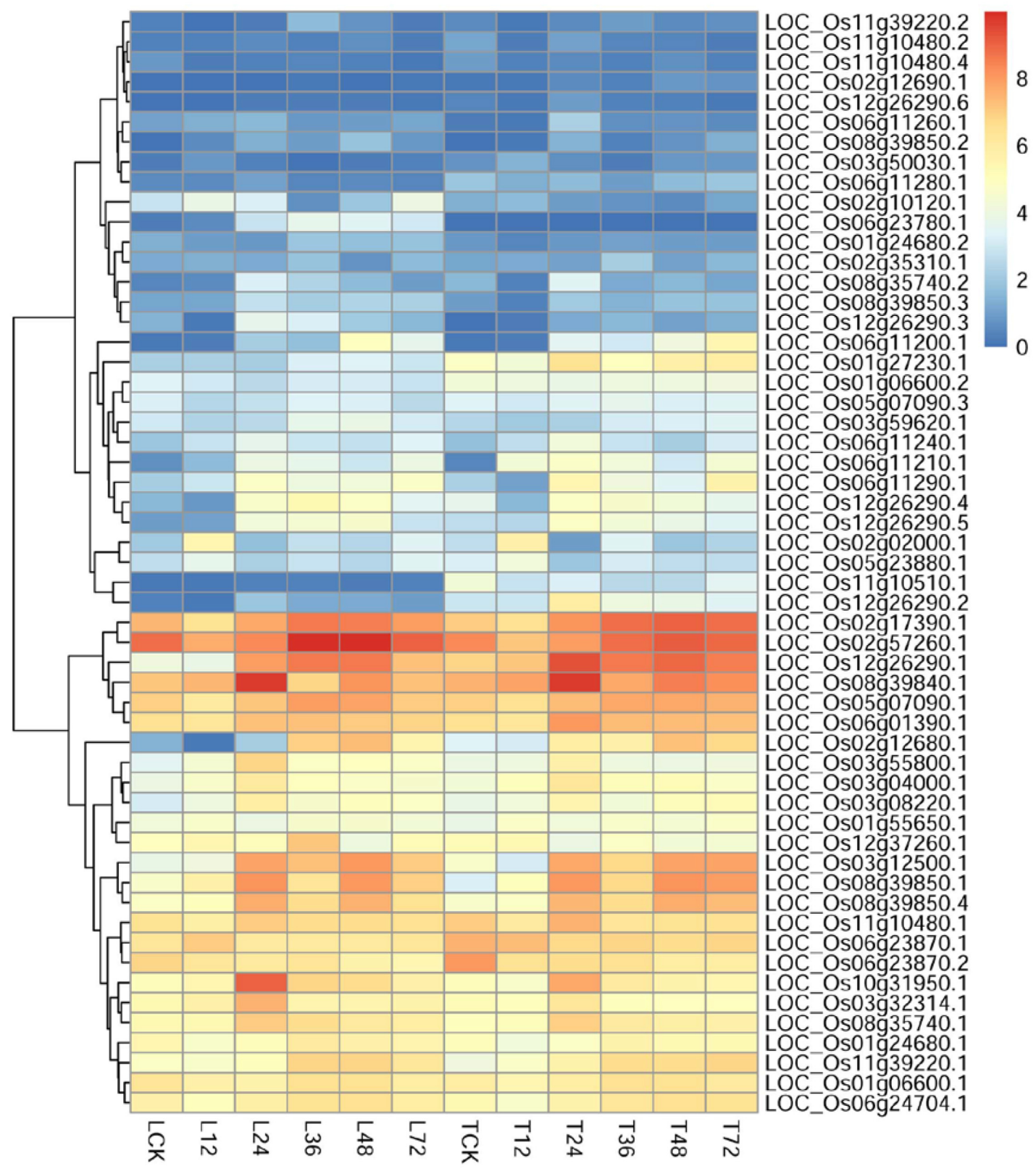

FIGURE 8 | Detailed expression profiles of genes related to alpha-linolenic acid metabolism.

and provides more effective protection to the photosynthetic apparatus than the Mehler reaction (Wu et al., 1991). $\mathrm{H}_{2} \mathrm{O}_{2}$ can trigger allergic reactions in plants, protecting healthy cells from infection through the programmed cell death of infected cells. The increased expression of genes involved in photorespiration metabolism can result in an increased $\mathrm{H}_{2} \mathrm{O}_{2}$ content in plants, which may improve plant disease resistance. One example is glycolate oxidase (GO), a key enzyme involved in plant photorespiration in the peroxisome. Research has found that GO is an alternative source for the production of $\mathrm{H}_{2} \mathrm{O}_{2}$ during both gene-for-gene and non-host resistance responses (Rojas and Mysore, 2012). In addition, serine hydroxymethyltransferase (SHMT) is an essential component of photorespiration. A previous study found that an osshm1 mutant exhibited chlorotic lesions and a lethal phenotype (Wang et al., 2015). Lin et al. (2012) found that OsCATC could increase hydrogen peroxide in leaves, which consequently promoted NO production and led to cell death. Thus, based on our gene expression results, we propose that photorespiration may play an important role after AG1 IA infection. Here, we have described the potential effects of photosynthesis and photorespiration on rice sheath blight resistance; however, further study is necessary 
to investigate how photosynthesis and photorespiration regulate sheath blight resistance.

\section{Plant-Pathogen Interactions}

Plants have established a series of defense mechanisms against pathogens during their co-evolution. The innate plant immune system can be generally thought of as having two layers. The first layer of immunity, known as PTI, is based on the recognition of PAMPs by cell surface pattern-recognition receptors, which confers resistance to most pathogenic microbes. The second layer of immunity primarily relies on the recognition of pathogensecreted effectors by plant-specific resistance proteins in direct or indirect ways, which leads to race-specific effector-triggered immunity (ETI) (Jones and Dangl, 2006). Chitin, a major component of fungal cell walls, is a representative PAMP. When a plant is attacked by a fungal pathogen, the pattern-recognition receptors located on the plasma membrane can recognize chitin and chitin oligosaccharide and trigger innate immune responses (Lenardon et al., 2010). CEBiP and CERK1 have been identified as critical components in chitin signaling in rice and Arabidopsis, respectively (Kaku et al., 2006; Lee et al., 2014). Here, we found that OsCERK1 exhibited a completely different trend in TeQing leaves at $12 \mathrm{~h}$, and we believe that OsCERK1 may participate in disease resistance in several different ways. Other genes, such as OsPtila, which has been shown to have an important role in regulating defense signaling for both $\mathrm{R}$ gene-mediated and basal resistance (Takahashi et al., 2007; Matsui et al., 2015) (Supplementary Table S8), increased in both TeQing and Lemont leaves but exhibited a more pronounced expression change in TeQing leaves at $24 \mathrm{~h}$.

Calcium, as an essential messenger, participates in the regulation of physiological metabolism through plant signal transduction pathways. Research has found that $\mathrm{Ca}^{2+}$ participates in oxidative burst signal transduction, regulates the expression of PR genes and mediates hypersensitive cell death (Torres and Dangl, 2006; Ogasawara et al., 2008; Du et al., 2009). Here, we observed the expression of genes involved in calcium-dependent signaling in plant-pathogen interactions in response to AG1 IA (Supplementary Table S8). One example is OsCPK10, a crucial regulator in plant immune responses that may regulate disease resistance by activating both SA- and JA-dependent defense responses ( $\mathrm{Fu}$ et al., 2013). We found that most of the genes in the calcium signaling pathway were down-regulated at $12 \mathrm{~h}$ and up-regulated at $24 \mathrm{~h}$. In addition, the degree of gene expression variability was higher in TeQing leaves than in Lemont leaves. Further research is needed to determine the effects of calcium on sheath blight resistance. Kinase cascades of the mitogen-activated protein kinase (MAPK) class play a critical role in the defense signaling evoked by the recognition of microbial-associated molecular patterns (MAMPs) that regulate several defense responses (Pitzschke et al., 2009). As shown in Supplementary Table S8, genes such as OsMKK1, OsMKK4, and OsMKK6 as well as other stress receptors exhibited changes in transcript levels in response to AG1 IA based on plant-pathogen interactions. Research has shown that OsMKK4-OsMPK3/OsMPK6 contribute substantially to the chitin elicitor-inducible biosynthesis of diterpenoid phytoalexins by regulating the expression of their biosynthetic genes (Yamane, 2013).

Members of the WRKY family are widely involved in plant defense responses, including positive and negative regulation of disease resistance (Pandey and Somssich, 2009). Here, we found that three WRKY transcription factors (WRKY24, WRKY53, and WRKY70) were expressed in both rice cultivars and that they were expressed at higher levels in TeQing leaves than in Lemont leaves. The expression level of WRKY70 increased at $12 \mathrm{~h}$ and reached a maximum at $24 \mathrm{~h}$ (Figure 6). Research has found that WRKY70 acts as an activator of SA-induced genes and a repressor of JA-responsive genes. The level of WRKY70 transcripts therefore reflects the cellular SA/JA balance and determines which type of response is favored ( $\mathrm{Li}$ et al., 2004). The PR1 gene is often used as a molecular marker of disease resistance. Research has shown that the expression of OsPR1a and OsPR1b increases after infection with Xanthomonas oryzae pv. oryzae (Xoo) and Magnaporthe oryzae (Wu et al., 2011). We found that the expression of these two genes in the two varieties was completely opposite (Figure 6). We believe that OsPR1a and OsPR1b may function in different pathways after AG1 IA infection.

\section{Changes in Phenylalanine Compounds}

Previous studies have shown that in the secondary metabolism of plant disease resistance, phenylpropanoid metabolism is an important metabolic pathway; this pathway includes flavonoids, stilbenes, monolignols, and various phenolic acids and participates in the formation of secondary resistance metabolites (Jones, 1984), such as phytoalexin (Dixon and Fuller, 1976), lignin (Legrand et al., 1976) and phenolic compounds (Glazener, 1982). KEGG results showed that phenylalanine metabolism plays an important role in TeQing leaves throughout the infection period, especially at $12 \mathrm{~h}$. Through gene analysis, a series of peroxidase (POD) genes and phenylalanine lyase (PAL) genes were found to be stimulated after AG1 IA inoculation. A previous study found that POD participates in the polymerization of monolignols into lignin and the reinforcement of the cell wall after pathogen attack (Marjamaa et al., 2009). Research has also shown that POD plays an important role in generating $\mathrm{H}_{2} \mathrm{O}_{2}$ as part of the defense response and confers resistance to a wide range of plant pathogens (Bindschedler et al., 2006). PAL plays a critical role in the phenylpropanoid pathway and has been reported to be responsive to pathogen attack (Huang et al., 2010). Research has shown that after pathogen infection, the activity of PAL exhibits dynamic changes over time (Nagarathna et al., 1993) and that PAL activity increased upon the development of $\mathrm{HR}$ in plant disease resistance (Southerton and Deverall, 1990). Here, nine differentially expressed PAL genes were identified and by compared the typical genes that participate in phenylalanine metabolic pathways. For example, LOC_Os06g44620.1 (Os4CL4), which encodes a 4-coumarate:coenzyme A ligase (4CL) and is thought to be involved in phenylalanine biosynthesis (Yoon et al., 2015), was more highly expressed in TeQing leaves than in Lemont leaves. The results suggest that PAL metabolism may play important roles at the initial infection stage. In addition, 3-dehydroquinate 
synthase, shikimate kinase, prephenate dehydratase and anthranilate synthase component I-1 were also found from this metabolic pathway. As shown in the current study, the phenylalanine pathway was up-regulated in TeQing leaves in the early stage $(12 \mathrm{~h})$ after AG1 IA infection. We speculated that at the initial stage of infection, TeQing leaves may prevent the encroachment of pathogens and protect the host cells by enhancing cell wall lignification and increasing other secondary metabolites. The difference between susceptible and resistant plants is associated with the ability of the plant to initiate defense processes effectively and in a timely manner. This is likely one of the reasons why the TeQing cultivar exhibits resistance to AG1 IA infection. Further research into these genes may provide candidates for the genetic improvement of rice and new insights into disease resistance.

\section{Changes in the JA and SA Pathways Related to AG1 IA Inoculation}

Jasmonic acid, a linolenic acid-derived cyclopentanone, plays regulatory roles in plant development and responses to fungal infection (Pieterse et al., 2009). Research has shown that JA signaling activates resistance to necrotrophic pathogens (Yang et al., 2013). Treating rice plants with JA induced the expression of a series of PR genes, suggesting that JA is involved in rice immunity to pathogens (Simmons et al., 1992; Mei et al., 2006). Our results indicated that the JA pathway is involved in the disease resistance to AG1 IA, which is in line with previous reports showing that the JA pathway mediates plant resistance to pathogens (Matić et al., 2016). In addition, as the infection time increased, genes involved in alpha-linolenic acid metabolism significantly enriched in Lemont leaves after $24 \mathrm{~h}$, and their expression suggested that the JA signaling pathway activation in response to AG1 IA is stronger in Lemont leaves. Furthermore, because JA synthesis-related genes were up-regulated at $12 \mathrm{~h}$, we speculate that the involvement of JA in the rice immune response may occur at the early stage of infection. The plant hormone SA is an important endogenous signal molecule in plant defense responses (Vlot et al., 2009). The SA response pathway is thought to be effective against biotrophic pathogens (not strictly distinguished) (Glazebrook, 2005). As important signal molecules in the plant defense response, SA- and JA-mediated pathways are relatively independent. However, SA-JA cross talk can also be a powerful mechanism used to prioritize one pathway over the other, depending on the sequence of events and type of threat encountered (Pieterse et al., 2012). We speculate that parasitic processes occur in the early stage of infection and, from the expression level of OsICS1, that SA is also involved in disease resistance.

\section{CONCLUSION}

In summary, the gene expression patterns in TeQing and Lemont leaves after AG1 IA infection provide a comprehensive overview of the transcriptome of two rice cultivars with contrasting disease responses. These patterns highlight the transcriptional variations between control and AG1 IA-infected plants. AG1
IA activated multiple resistance pathways, and DEGs were involved in the defense response, signal transduction and other processes, suggesting that pathogen response is regulated by multi-gene networks. A series of genes involved in disease-related metabolic pathways were significantly regulated, demonstrating that AG1 IA infection negatively affects the growth of rice. In addition, we identified some metabolic pathways that were common to both the TeQing and Lemont cultivars. We believe that the biosynthesis of JA, the basic plant metabolic processes of photosynthesis and photorespiration, and the synthesis of phenylalanine compounds may be important for disease tolerance in rice. Further research is necessary to identify candidate genes to determine whether these DEGs are necessary for the differences associated with AG1 IA infection between TeQing and Lemont plants. The data obtained in this study may be used to identify the most suitable candidate genes for carrying out genetic modification of susceptible rice cultivars to generate disease-tolerant rice cultivars.

\section{AVAILABILITY OF SUPPORTING DATA}

The raw sequence data supporting the results of this article are available in the Short Read Archive (SRA) (accession number SRP113646). https://www.ncbi.nlm.nih.gov/Traces/study/?acc= SRP113646

\section{AUTHOR CONTRIBUTIONS}

JiZ completed the majority of the work for this article, including the experimental design, data analysis, and drafting of the manuscript. LC and CF provided useful suggestions. JiZ and YC carried out bioinformatic analyses. PL, LW, HL, SL, QD, SW, JuZ, YL, and AZ revised the manuscript. All authors read and approved the final manuscript.

\section{FUNDING}

This work was supported by the National 973 Project (2014CB160304) and the National Natural Science Foundation (31400130).

\section{ACKNOWLEDGMENT}

The authors thank all contributors for their work and would like to thank the reviewers for their valuable comments and suggestions.

\section{SUPPLEMENTARY MATERIAL}

The Supplementary Material for this article can be found online at: http://journal.frontiersin.org/article/10.3389/fpls.2017.01422/ full\#supplementary-material 


\section{REFERENCES}

Anderson, P. K., Cunningham, A. A., Patel, N. G., Morales, F. J., Epstein, P. R., and Daszak, P. (2004). Emerging infectious diseases of plants: pathogen pollution, climate change and agrotechnology drivers. Trends Ecol. Evol. 19, 535-544. doi: 10.1016/j.tree.2004.07.021

Andolfo, G., and Ercolano, M. R. (2015). Plant innate immunity multicomponent model. Front. Plant Sci. 6:987. doi: 10.3389/fpls.2015.00987

Bagnaresi, P., Biselli, C., Orrù, L., Urso, S., Crispino, L., Abbruscato, P., et al. (2012). Comparative transcriptome profiling of the early response to Magnaporthe oryzae in durable resistant vs susceptible rice (Oryza sativa L.) genotypes. PLoS ONE 7:e51609. doi: 10.1371/journal.pone.0051609

Bindschedler, L. V., Dewdney, J., Blee, K. A., Stone, J. M., Asai, T., Plotnikov, J., et al. (2006). Peroxidase-dependent apoplastic oxidative burst in Arabidopsis required for pathogen resistance. Plant J. 47, 851-863. doi: 10.1111/j.1365313X.2006.02837.X

Boller, T., and He, S. Y. (2009). Innate immunity in plants: an arms race between pattern recognition receptors in plants and effectors in microbial pathogens. Science 324, 742-744. doi: 10.1126/science.1171647

Bolton, M. D. (2009). Primary metabolism and plant defense-fuel for the fire. Mol. Plant Microbe Interact. 22, 487-497. doi: 10.1094/MPMI-22-5-0487

Bolton, M. D., Kolmer, J. A., Xu, W. W., and Garvin, D. F. (2008). Lr34-mediated leaf rust resistance in wheat: transcript profiling reveals a high energetic demand supported by transient recruitment of multiple metabolic pathways. Mol. Plant Microbe Interact. 21, 1515-1527. doi: 10.1094/MPMI-21-12-1515

Brooks, S. A. (2007). Sensitivity to a phytotoxin from Rhizoctonia solani correlates with sheath blight susceptibility in rice. Phytopathology 97, 1207-1212. doi: 10.1094/PHYTO-97-10-1207

Buchanan, B. B., and Balmer, Y. (2005). Redox regulation: a broadening horizon. Annu. Rev. Plant Biol. 56, 187-220. doi: 10.1146/annurev.arplant.56.032604. 144246

Chang, R. I., Chu, C. C., Wu, Y. Y., and Chen, Y. L. (2010). Gene clustering by using query-based self-organizing maps. Expert Syst. Appl. 37, 6689-6694. doi: 10.1016/j.eswa.2010.03.050

Chao, J., Jin, J., Wang, D., Han, R., Zhu, R., Zhu, Y., et al. (2014). Cytological and transcriptional dynamics analysis of host plant revealed stage-specific biological processes related to compatible rice-Ustilaginoidea virens interaction. PLoS ONE 9:e91391. doi: 10.1371/journal.pone.0091391

Coram, T. E., Wang, M. L., and Chen, X. (2008). Transcriptome analysis of high temperature adult plant resistance conditioned by Yr39 during wheat-Puccinia striiformis f. sp. tritici interaction. Mol. Plant Pathol. 9, 479-493. doi: 10.1111/j. 1364-3703.2008.00476.x

Ditt, R. F., Kerr, K. F., De, F. P., Delrow, J., Comai, L., and Nester, E. W. (2006). The Arabidopsis thaliana transcriptome in response to Agrobacterium tumefaciens. Mol. Plant Microbe Interact. 19, 665-681. doi: 10.1094/MPMI-190665

Dixon, R. A., and Fuller, K. W. (1976). Effects of synthetic auxin levels on phaseolin production and phenylalanine ammonia-lyase (PAL) activity in tissue cultures of Phaseolus vulgaris L. Physiol. Plant Pathol. 9, 299-312. doi: 10.1016/00484059(76)90063- 1

Dodds, P. N., and Rathjen, J. P. (2010). Plant immunity: towards an integrated view of plant-pathogen interactions. Nat. Rev. Genet. 11, 539-548. doi: 10.1038/ $\operatorname{nrg} 2812$

Du, L., Ali, G. S., Simons, K. A., Hou, J., Yang, T., Reddy, A. S., et al. (2009). $\mathrm{Ca}^{2+} /$ calmodulin regulates salicylic-acid-mediated plant immunity. Nature 457, 1154-1158. doi: 10.1038/nature07612

Fu, L., Yu, X., and An, C. (2013). Overexpression of constitutively active OsCPK10 increases Arabidopsis resistance against Pseudomonas syringae pv. tomato and rice resistance against Magnaporthe grisea. Plant Physiol. Biochem. 73, 202-210. doi: 10.1016/j.plaphy.2013.10.004

Gechev, T. S., Van Breusegem, F., Stone, J. M., Denev, I., and Laloi, C. (2006). Reactive oxygen species as signals that modulate plant stress responses and programmed cell death. Bioessays 28, 1091-1101. doi: 10.1002/bies.20493

Glazebrook, J. (2005). Contrasting mechanisms of defense against biotrophic and necrotrophic pathogens. Annu. Rev. Phytopathol. 43, 205-227. doi: 10.1146/ annurev.phyto.43.040204.135923

Glazener, J. A. (1982). Accumulation of phenolic compounds in cells and formation of lignin-like polymers in cell walls of young tomato fruits after inoculation with
Botrytis cinerea. Physiol. Plant. Pathol. 20, 11-25. doi: 10.1016/0048-4059(82) 90019-4

Göhre, V., Jones, A. M., Sklenř, J., Robatzek, S., and Weber, A. P. (2012). Molecular crosstalk between PAMP-triggered immunity and photosynthesis. Mol. Plant Microbe Interact. 25, 1083-1092. doi: 10.1094/MPMI-11-11-0301

Hammerschmidt, R. (1999). Phytoalexins: what have we learned after 60 years? Annu. Rev. Phytopathol. 37, 285-306. doi: 10.1146/annurev.phyto.37.1.285

Huang, J., Gu, M., Lai, Z., Fan, B., Shi, K., Zhou, Y. H., et al. (2010). Functional analysis of the Arabidopsis $P A L$ gene family in plant growth, development, and response to environmental stress. Plant Physiol. 153, 1526-1538. doi: 10.1104/ pp. 110.157370

Jain, M. (2012). Next-generation sequencing technologies for gene expression profiling in plants. Brief. Funct. Genomics 11, 63-70. doi: 10.1093/bfgp/elr038

Jia, Y., Correavictoria, F., Mcclung, A., Zhu, L., Liu, G., Wamishe, Y., et al. (2007). Rapid determination of rice cultivar responses to the sheath blight pathogen Rhizoctonia solani using a micro-chamber screening method. Plant Dis. 91, 485-489. doi: 10.1094/PDIS-91-5-0485

Jones, D. H. (1984). Phenylalanine ammonia-lyase: regulation of its induction, and its role in plant development. Phytochemistry 23, 1349-1359. doi: 10.1016/ S0031-9422(00)80465-3

Jones, J. D., and Dangl, J. L. (2006). The plant immune system. Nature 444, 323-329. doi: 10.1038/nature05286

Kaku, H., Nishizawa, Y., Ishii-Minami, N., Akimoto-Tomiyama, C., Dohmae, N., Takio, K., et al. (2006). Plant cells recognize chitin fragments for defense signaling through a plasma membrane receptor. Proc. Natl. Acad. Sci. U.S.A. 103, 11086-11091. doi: 10.1073/pnas.0508882103

Kangasjärvi, S., Neukermans, J., Li, S., Aro, E. M., and Noctor, G. (2012). Photosynthesis, photorespiration, and light signalling in defence responses. J. Exp. Bot. 63, 1619-1636. doi: 10.1093/jxb/err402

Kawahara, Y., Oono, Y., Kanamori, H., Matsumoto, T., Itoh, T., and Minami, E. (2012). Simultaneous RNA-seq analysis of a mixed transcriptome of rice and blast fungus interaction. PLOS ONE 7:e49423. doi: 10.1371/journal.pone. 0049423

Kerchev, P. I., Fenton, B., Foyer, C. H., and Hancock, R. D. (2012). Plant responses to insect herbivory: interactions between photosynthesis, reactive oxygen species and hormonal signalling pathways. Plant Cell Environ. 35, 441-453. doi: 10.1111/j.1365-3040.2011.02399.x

Kereiche, S., Kiss, A. Z., Kouril, R., Boekema, E. J., and Horton, P. (2009). The PsbS protein controls the macro-organisation of photosystem II complexes in the grana membranes of higher plant chloroplasts. FEBS Lett. 584, 759-764. doi: 10.1016/j.febslet.2009.12.031

Kim, D., Pertea, G., Trapnell, C., Pimentel, H., Kelley, R., and Salzberg, S. L. (2013). TopHat2: accurate alignment of transcriptomes in the presence of insertions, deletions and gene fusions. Genome Biol. 14:R36. doi: 10.1186/gb-2013-144-r36

Lai, Z., and Mengiste, T. (2013). Genetic and cellular mechanisms regulating plant responses to necrotrophic pathogens. Curr. Opin. Plant Biol. 16, 505-512. doi: 10.1016/j.pbi.2013.06.014

Lee, J., Bricker, T. M., Lefevre, M., Pinson, S. R. M., and Oard, J. H. (2006). Proteomic and genetic approaches to identifying defence-related proteins in rice challenged with the fungal pathogen Rhizoctonia solani. Mol. Plant Pathol. 7, 405-416. doi: 10.1111/j.1364-3703.2006.00350.x

Lee, W. S., Rudd, J. J., Hammond-Kosack, K. E., and Kanyuka, K. (2014). Mycosphaerella graminicola LysM effector-mediated stealth pathogenesis subverts recognition through both CERK1 and CEBiP homologues in wheat. Mol. Plant Microbe Interact. 27, 236-243. doi: 10.1094/MPMI-07-13-0201-R

Legrand, M., Fritig, B., and Hirth, L. (1976). Enzymes of the phenylpropanoid pathway and the necrotic reaction of hypersensitive tobacco to tobacco mosaic virus. Phytochemistry 15, 1353-1359. doi: 10.1016/S0031-9422(00)97117-6

Lenardon, M. D., Munro, C. A., and Gow, N. A. R. (2010). Chitin synthesis and fungal pathogenesis. Curr. Opin. Microbiol. 13, 416-423. doi: 10.1016/j.mib. 2010.05 .002

Li, H., Handsaker, B., Wysoker, A., Fennell, T., Ruan, J., Homer, N., et al. (2009). The sequence alignment/map format and SAMtools. Bioinformatics 25, 2078-2079. doi: 10.1093/bioinformatics/btp352

Li, J., Brader, G., and Palva, E. T. (2004). The WRKY70 transcription factor: a node of convergence for jasmonate-mediated and salicylate-mediated signals in plant defense. Plant Cell 16, 319-331. doi: 10.1105/tpc.016980 
Li, S., Gao, F., Xie, K., Zeng, X., Cao, Y., Zeng, J., et al. (2016). The OsmiR396cOsGRF4-OsGIF1 regulatory module determines grain size and yield in rice. Plant Biotechnol. J. 14, 2134-2146. doi: 10.1111/pbi.12569

Lin, A., Wang, Y., Tang, J., Xue, P., Li, C., Liu, L., et al. (2012). Nitric oxide and protein S-nitrosylation are integral to hydrogen peroxide-induced leaf cell death in rice. Plant Physiol. 158, 451-464. doi: 10.1104/pp.111.184531

Liu, G., Jia, Y., Correa-Victoria, F. J., Prado, G. A., Yeater, K. M., McClung, A., et al. (2009). Mapping quantitative trait loci responsible for resistance to sheath blight in rice. Phytopathology 99, 1078-1084. doi: 10.1094/PHYTO-99-9-1078

Liu, G., Jia, Y., Mcclung, A., Oard, J. H., Lee, F. N., and Correll, J. C. (2013). Confirming QTLs and finding additional loci responsible for resistance to rice sheath blight disease. Plant Dis. 97, 113-117. doi: 10.1270/jsbbs.65.170

Marchetti, M. A., and Bollich, C. N. (1991). Quantification of the relationship between sheath blight severity and yield loss in rice. Plant Dis. 75, 773-775. doi: 10.1094/PD-75-0773

Marjamaa, K., Kukkola, E. M., and Fagerstedt, K. V. (2009). The role of xylem class III peroxidases in lignification. J. Exp. Bot. 60, 367-376. doi: 10.1093/jxb/ern278

Marshall, D. S., and Rush, M. C. (1980). Infection cushion formation on rice sheaths by Rhizoctonia solani. Phytopathology 70, 947-950. doi: 10.1094/Phyto70-947

Maruthasalam, S., Kalpana, K., Kumar, K. K., Loganathan, M., Poovannan, K., Raja, J. A., et al. (2007). Pyramiding transgenic resistance in elite indica rice cultivars against the sheath blight and bacterial blight. Plant Cell Rep. 26, 791-804. doi: 10.1007/s00299-006-0292-5

Matić, S., Bagnaresi, P., Biselli, C., Orru’, L., Amaral Carneiro, G., Siciliano, I., et al. (2016). Comparative transcriptome profiling of resistant and susceptible rice genotypes in response to the seedborne pathogen Fusarium fujikuroi. BMC Genomics 17:608. doi: 10.1186/s12864-016-2925-6

Matsui, H., Takahashi, A., and Hirochika, H. (2015). Rice immune regulator, OsPtila, is specifically phosphorylated at the plasma membrane. Plant Signal. Behav. 10:e991569. doi: 10.4161/15592324.2014.991569

Mei, C., Qi, M., Sheng, G., and Yang, Y. (2006). Inducible overexpression of a rice allene oxide synthase gene increases the endogenous jasmonic acid level, PR gene expression, and host resistance to fungal infection. Mol. Plant Microbe Interact. 19, 1127-1137. doi: 10.1094/MPMI-19-1127

Müller, P., Li, X. P., and Niyogi, K. K. (2001). Non-photochemical quenching. A response to excess light energy. Plant Physiol. 125, 1558-1566. doi: 10.1104/ pp.125.4.1558

Nagarathna, K. C., Shetty, S. A., and Shetty, H. S. (1993). Phenylalanine ammonia lyase activity in pearl millet seedlings and its relation to downy mildew disease resistance. J. Exp. Bot. 44, 1291-1296. doi: 10.1093/jxb/44.8.1291

Nakashima, K., and Yamaguchi-Shinozaki, K. (2009). Transcriptional regulatory networks in response to abiotic stresses in Arabidopsis and grasses. Plant Physiol. 149, 88-95. doi: 10.1104/pp.108.129791

Niyogi, K. K., Li, X. P., Rosenberg, V., and Jung, H. S. (2005). Is PsbS the site of non-photochemical quenching in photosynthesis? J. Exp. Bot. 56, 375-382. doi: $10.1093 / \mathrm{jxb} / \mathrm{eri056}$

Ogasawara, Y., Kaya, H., Hiraoka, G., Yumoto, F., Kimura, S., Kadota, Y., et al. (2008). Synergistic activation of the Arabidopsis NADPH oxidase AtrbohD by $\mathrm{Ca}^{2+}$ and phosphorylation. J. Biol. Chem. 283, 8885-8892. doi: 10.1074/jbc. M708106200

Okubara, P. A., Dickman, M. B., and Blechl, A. E. (2014). Molecular and genetic aspects of controlling the soilborne necrotrophic pathogens Rhizoctonia and Pythium. Plant Sci. 228, 61-70. doi: 10.1016/j.plantsci.2014.02.001

Pandey, S. P., and Somssich, I. E. (2009). The role of WRKY transcription factors in plant immunity. Plant Physiol. 150, 1648-1655. doi: 10.1104/pp.109.138990

Pieterse, C. M., Van der Does, D., Zamioudis, C., Leon-Reyes, A., and Van Wees, S. C. (2012). Hormonal modulation of plant immunity. Annu. Rev. Cell Dev. 28, 489-521. doi: 10.1146/annurev-cellbio-092910-154055

Pieterse, C. M. J., Leon-Reyes, A., van der Ent, S., and Van Wees, S. C. M. (2009). Networking by small-molecules hormones in plant immunity. Nat. Chem. Biol. 5, 308-316. doi: 10.1038/nchembio.164

Pinson, S. R. M., Capdevielle, F. M., and Oard, J. H. (2005). Confirming QTLs and finding additional loci conditioning sheath blight resistance in rice using recombinant inbred lines. Crop Sci. 45, 503-510. doi: 10.2135/cropsci2005.0503

Pitzschke, A., Schikora, A., and Hirt, H. (2009). MAPK cascade signalling networks in plant defence. Curr. Opin. Plant Biol. 12, 421-426. doi: 10.1016/j.pbi.2009. 06.008
Que, Y., Su, Y., Guo, J., Wu, Q., and Xu, L. (2014). A global view of transcriptome dynamics during Sporisorium scitamineum challenge in sugarcane by RNA-Seq. PLoS ONE 9:e106476. doi: 10.1371/journal.pone.0106476

Rojas, C. M., and Mysore, K. S. (2012). Glycolate oxidase is an alternative source for $\mathrm{H}_{2} \mathrm{O}_{2}$ production during plant defense responses and functions independently from NADPH oxidase. Plant Signal. Behav. 7, 752-755. doi: 10.4161/psb. 20429

Rojas, C. M., Senthilkumar, M., Tzin, V., and Mysore, K. S. (2014). Regulation of primary plant metabolism during plant-pathogen interactions and its contribution to plant defense. Front. Plant Sci. 5:17. doi: 10.3389/fpls.2014. 00017

Schaller, A., and Stintzi, A. (2009). Enzymes in jasmonate biosynthesis - structure, function, regulation. Phytochemistry 70, 1532-1538. doi: 10.1016/j.phytochem. 2009.07.032

Shu, C. W., Chen, J. L., Si, S., Zhang, M. L., Wang, C. J. Z., and Zhou, E. X. (2015). Two distinct classes of protein related to GTB and RRM are critical in the sclerotial metamorphosis process of Rhizoctonia solani AG-1 IA. Funct. Integr. Genomics 15, 449-459. doi: 10.1007/s10142-015-0435-2

Silva, J., Scheffler, B., Sanabria, Y., Guzman, C. D., Galam, D., Farmer, A., et al. (2012). Identification of candidate genes in rice for resistance to sheath blight disease by whole genome sequencing. Theor. Appl. Genet. 124, 63-74. doi: 10.1007/s00122-011-1687-4

Simmons, C. R., Litts, J. C., Huang, N., and Rodriguez, R. L. (1992). Structure of a rice beta-glucanase gene regulated by ethylene, cytokinin, wounding, salicylic acid and fungal elicitors. Plant Mol. Biol. 18, 33-45. doi: 10.1007/BF00018454

Skibbe, D. S., Doehlemann, G., Fernandes, J., and Walbot, V. (2010). Maize tumors caused by Ustilago maydis require organ-specific genes in host and pathogen. Science 328, 89-92. doi: 10.1126/science.1185775

Southerton, S. G., and Deverall, B. J. (1990). Changes in phenylalanine ammonialyase and peroxidase activities in wheat cultivars expressing resistance to the leaf-rust fungus. Plant Pathol. 39, 223-230. doi: 10.1111/j.1365-3059.1990. tb02496.x

Takahashi, A., Agrawal, G. K., Yamazaki, M., Onosato, K., Miyao, A., Kawasaki, T., et al. (2007). Rice Ptila negatively regulates RAR1-dependent defense responses. Plant Cell 19, 2940-2951. doi: 10.1105/tpc.106.047142

Torres, M. A., and Dangl, J. L. (2006). Reactive oxygen species signaling in response to pathogens. Plant Physiol. 141, 373-378. doi: 10.1104/pp.106.079467

Trapnell, C., Williams, B. A., Pertea, G., Mortazavi, A., Kwan, G., van Baren, M. J., et al. (2010). Transcript assembly and quantification by RNA-Seq reveals unannotated transcripts and isoform switching during cell differentiation. Nat. Biotechnol. 28, 511-515. doi: 10.1038/nbt.1621

Venu, R. C., Jia, Y., Gowda, M., Jia, M. H., Jantasuriyarat, C., Stahlberg, E., et al. (2007). RL-SAGE and microarray analysis of the rice transcriptome after Rhizoctonia solani infection. Mol. Genet. Genomics 278, 421-431. doi: 10.1007/ s00438-007-0260-y

Vidhyasekaran, P., Ponmalar, T. R., Samiyappan, R., Velazhahan, R., Vimala, R., Ramanathan, A., et al. (1997). Host-specific toxin production by Rhizoctonia solani, the rice sheath blight pathogen. Phytopathology 87, 1258-1263. doi: 10.1094/PHYTO.1997.87.12.1258

Vlot, A. C., Dempsey, D. A., and Klessig, D. F. (2009). Salicylic acid, a multifaceted hormone to combat disease. Annu. Rev. Phytopathol. 47, 177-206. doi: 10.1146/ annurev.phyto.050908.135202

Wang, C., Zien, C. A., Afitlhile, M., Welti, R., Hildebrand, D. F., and Wang, X. (2000). Involvement of phospholipase D in wound-induced accumulation of jasmonic acid in Arabidopsis. Plant Cell 12, 2237-2246. doi: 10.1105/tpc.12.11. 2237

Wang, D., Liu, H., Li, S., Zhai, G., Shao, J., and Tao, Y. (2015). Characterization and molecular cloning of a serine hydroxymethyltransferase 1 (OsSHM1) in rice. J. Integr. Plant Biol. 57, 745-756. doi: 10.1111/jipb.12336

Wang, Y., Kwon, S. J., Wu, J., Choi, J., Lee, Y. H., Agrawal, G. K., et al. (2014). Transcriptome analysis of early responsive genes in rice during Magnaporthe oryzae infection. Plant Pathol. J. 30, 343-354. doi: 10.5423/PPJ.OA.06.2014. 0055

Wang, Z., Gerstein, M., and Snyder, M. (2009). RNA-Seq: a revolutionary tool for transcriptomics. Nat. Rev. Genet. 10, 57-63. doi: 10.1038/nrg2484

Wildermuth, M. C., Dewdney, J., Wu, G., and Ausubel, F. M. (2001). Isochorismate synthase is required to synthesize salicylic acid for plant defence. Nature 414, 562-565. doi: $10.1038 / 35107108$ 
Wu, J., Neimanis, S., and Heber, U. (1991). Photorespiration is more effective than the mehler reaction in protecting the photosynthetic apparatus against photoinhibition. Bot. Acta 104, 283-291. doi: 10.1111/j.1438-8677.1991. tb00231.x

Wu, Q., Hou, M. M., Li, L. Y., Liu, L. J., Hou, Y. X., and Liu, G. Z. (2011). Induction of pathogenesis-related proteins in rice bacterial blight resistant gene Xa21mediated interactions with Xanthomonas oryzae pv. oryzae. J. Plant Pathol. 93 , 455-459.

Yamane, H. (2013). Biosynthesis of phytoalexins and regulatory mechanisms of it in rice. Biosci. Biotechnol. Biochem. 77, 1141-1148. doi: 10.1271/bbb.130109

Yang, D. L., Yang, Y., and He, Z. (2013). Roles of plant hormones and their interplay in rice immunity. Mol. Plant 6, 675-685. doi: 10.1093/mp/sst056

Yang, L., Xie, L., Xue, B., Goodwin, P. H., Quan, X., Zheng, C., et al. (2015). Comparative transcriptome profiling of the early infection of wheat roots by Gaeumannomyces graminis var. tritici. PLoS ONE 10:e0120691. doi: 10.1371/ journal.pone.0120691

Yoon, J., Choi, H., and An, G. (2015). Roles of lignin biosynthesis and regulatory genes in plant development. J. Integr. Plant Biol. 57, 902-912. doi: 10.1111/jipb. 12422

Zhang, J., Simmons, C., Yalpani, N., Crane, V., Wilkinson, H., and Kolomiets, M. (2005). Genomic analysis of the 12-oxo-phytodienoic acid reductase gene family of Zea mays. Plant Mol. Biol. 59, 323-343. doi: 10.1007/s11103-0058883-z

Zhao, C. J., Wang, A. R., Shi, Y. J., Wang, L. Q., Liu, W. D., Wang, Z. H., et al. (2008). Identification of defense-related genes in rice responding to challenge by Rhizoctonia solani. Theor. Appl. Genet. 116, 501-516. doi: 10.1007/s00122007-0686-y

Zheng, A., Lin, R., Zhang, D., Qin, P., Xu, L., Ai, P., et al. (2013). The evolution and pathogenic mechanisms of the rice sheath blight pathogen. Nat. Commun. 4, 273-275. doi: 10.1038/ncomms 2427

Conflict of Interest Statement: The authors declare that the research was conducted in the absence of any commercial or financial relationships that could be construed as a potential conflict of interest.

Copyright (c) 2017 Zhang, Chen, Fu, Wang, Liu, Cheng, Li, Deng, Wang, Zhu, Liang, Li and Zheng. This is an open-access article distributed under the terms of the Creative Commons Attribution License (CC BY). The use, distribution or reproduction in other forums is permitted, provided the original author(s) or licensor are credited and that the original publication in this journal is cited, in accordance with accepted academic practice. No use, distribution or reproduction is permitted which does not comply with these terms. 